\title{
Total Synthesis and Stereochemical Reassignment of Citrafungin A.
}

\author{
Zongjia Chen, Angus Robertson, Jonathan M. White and Mark A. Rizzacasa.* \\ School of Chemistry and Bio21 Molecular Science and Biotechnology Institute, The University of \\ Melbourne, Melbourne, Victoria, 3010, Australia.
}

\section{Experimental Section}

\section{Supporting Information}

\section{General}

Preparation of cyclobutene diester $\mathbf{1 0}$

Preparation of lactone 12

Preparation of triester 11

Preparation of tosylate

Preparation of alkene 13

Preparation of silyl ether 14

Preparation of alkyne 8

Preparation of allylic alcohol 16

Preparation of lactone 17

Preparation of vinyl iodide $\mathbf{2 0}$

Kishi-Nozaki coupling: preparation of undesired lactone $\mathbf{2 1}$

Preparation of diacid ent-3

Preparation of citrafungin A tetra-t-butyl ester (19)

Preparation of citrafungin A (1a)

Table 1: NMR data for natural and synthetic 1a

Table: ${ }^{13} \mathrm{C}$ NMR chemical shift differences for natural and synthetic 1a and $\mathbf{1}$

References 


\section{General}

All reactions were performed under an inert argon or nitrogen atmosphere in flame-dried or ovendried $\left(150{ }^{\circ} \mathrm{C}\right)$ glassware. Anhydrous tetrahydrofuran (THF), diethyl ether $\left(\mathrm{Et}_{2} \mathrm{O}\right)$ and dichloromethane (DCM) were obtained from a solvent dispensing system where solvents were dried by passage through two packed columns of neutral alumina under argon. Dry methanol $(\mathrm{MeOH})$ was distilled from magnesium methoxide or $\mathrm{CaSO}_{4}$ and stored over $4 \AA$ molecular sieves. Dry triethylamine $\left(\mathrm{NEt}_{3}\right)$ and acetonitrile $(\mathrm{MeCN})$ were freshly distilled from calcium hydride under nitrogen atmosphere. Diisopropylamine (DIPA) was distilled from $\mathrm{NaOH}$ under nitrogen or argon atmosphere immediately prior to use. Hexamethylphosphoramide (HMPA) and $\mathrm{N}, \mathrm{N}$ dimethylformamide (DMF) were dried by storage over $4 \AA$ molecule sieves. All other commercial reagents were used as received. Brine refers to a saturated aqueous solution of $\mathrm{NaCl}$ and petrol refers to petroleum spirits of the fraction boiling between 40 and $60{ }^{\circ} \mathrm{C}$. Flash chromatography was carried out using silica gel 60. Analytical thin layer chromatography (TLC) was conducted on aluminium backed plates ( $2 \mathrm{~mm}$ silica gel $60 \mathrm{~F}_{254}$ ) and chromatograms were visualised under UV light (365 nm) and with solutions of $20 \% \mathrm{w} / \mathrm{w}$ phosphomolybdic acid in ethanol (PMA), 20\% w/w potassium permanganate in water (PP) or $5 \% \mathrm{w} / \mathrm{v}$ cerium (IV) ammonium molybdate and $1 \% \mathrm{w} / \mathrm{v}$ ceric sulphate in dilute sulphuric acid (CAM). Melting points were obtained in open capillaries and are uncorrected. Optical rotations were recorded at $22{ }^{\circ} \mathrm{C}$ in a $10.0 \mathrm{~cm}$ microcell and units are deg. $\mathrm{cm}^{2} \mathrm{~g}^{-1}$. Infrared (IR) spectra were recorded using an attenuated total reflectance (ATR) attachment. High-resolution mass spectra (HRMS) were obtained using electrospray ionisation (ESI). Nuclear magnetic resonance (NMR) spectra were recorded at 400,500 or $600 \mathrm{MHz}$ and chemical sifts $(\delta)$ were internally referenced to the residual proton resonance $\mathrm{CDCl}_{3}(\delta 7.26 \mathrm{ppm}), \mathrm{CD}_{3} \mathrm{CN}$ and $\mathrm{CD}_{3} \mathrm{OD}(\delta 3.31 \mathrm{ppm})$.

\section{Cyclobutene diester 10}

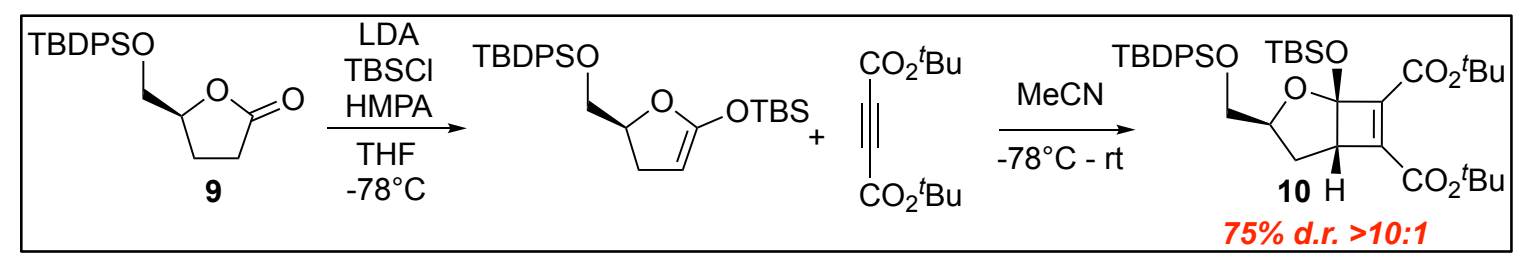

To a solution of diisopropylamine $(4.2 \mathrm{~mL}, 30.2 \mathrm{mmol})$ in THF $(8 \mathrm{~mL})$ at $-78{ }^{\circ} \mathrm{C}$ was added ${ }^{n} \mathrm{BuLi}$ (2.5 $\mathrm{M}$ in hexanes, $11.3 \mathrm{~mL}, 28.2 \mathrm{mmol}$ ) and the resultant solution was warmed to $0^{\circ} \mathrm{C}$ for 10 minutes. The solution was cooled again to $-78^{\circ} \mathrm{C}$ and a solution of lactone $9(5 \mathrm{~g}, 14.1 \mathrm{mmol})$ in $\mathrm{THF}(10 \mathrm{~mL})$ was added dropwise $(1.0 \mathrm{~mL} / \mathrm{min})$ and the resultant mixture stirred at $-78^{\circ} \mathrm{C}$ for 1.5 hours. A solution of TBSCl $(2.76 \mathrm{~g}, 18.3 \mathrm{mmol})$ and HMPA $(2 \mathrm{~mL})$ in THF $(10 \mathrm{~mL})$ was then added dropwise $(1.0$ $\mathrm{mL} / \mathrm{min}$ ) and the reaction mixture stirred for an additional $30 \mathrm{mins}$ at $-78^{\circ} \mathrm{C}$ then $1 \mathrm{~h}$ at $\mathrm{rt}$. The mixture was then cooled to $0{ }^{\circ} \mathrm{C}$, diluted with hexane $(25 \mathrm{~mL})$, quenched with water $(25 \mathrm{~mL})$ and the organic layer was washed with water, brine then dried over $\mathrm{Na}_{2} \mathrm{SO}_{4}$ and concentrated to afford the crude silyl ketene acetal, which was immediately dissolved in $\mathrm{CH}_{2} \mathrm{Cl}_{2}(20 \mathrm{~mL})$ and cooled to $-78{ }^{\circ} \mathrm{C}$. Di-tertbutyl but-2-ynedioate $(3.19 \mathrm{~g}, 14.1 \mathrm{mmol})$ was added and the solution allowed to warm to rt and stirred overnight. The solvent was removed under reduced pressure and purification by flash chromatography with 5\% EtOAc/petrol as eluent to afford the cyclobutene $\mathbf{1 0}(7.35 \mathrm{~g}, 75 \%)$, as a yellow oil. $[\alpha]_{\mathrm{D}}=-12.8\left(c 1.15, \mathrm{CH}_{2} \mathrm{Cl}_{2}\right) .{ }^{1} \mathrm{H} \mathrm{NMR}\left(400 \mathrm{MHz}, \mathrm{CDCl}_{3}\right) \delta 0.12(\mathrm{~s}, 3 \mathrm{H}), 0.19(\mathrm{~s}, 3 \mathrm{H})$, $0.90(\mathrm{~s}, 9 \mathrm{H}), 1.06(\mathrm{~s}, 9 \mathrm{H}), 1.51(\mathrm{~s}, 9 \mathrm{H}), 1.52(\mathrm{~s}, 9 \mathrm{H}), 1.72(\mathrm{~m}, 1 \mathrm{H}), 1.91(\mathrm{dd}, J=12.9,5.1 \mathrm{~Hz}, 1 \mathrm{H})$, $3.21(\mathrm{~d}, J=7.6 \mathrm{~Hz}, 1 \mathrm{H}), 3.91-3.80(\mathrm{~m}, 2 \mathrm{H}), 4.03(\mathrm{dq}, J=9.6,4.7 \mathrm{~Hz}, 1 \mathrm{H}), 7.47-7.33(\mathrm{~m}, 6 \mathrm{H}), 7.69$ $(\mathrm{m}, 4 \mathrm{H}) .{ }^{13} \mathrm{C} \mathrm{NMR}\left(101 \mathrm{MHz}, \mathrm{CDCl}_{3}\right) \delta-3.3,-2.9,17.9,19.4,25.8,27.0,28.0,28.2,28.2,28.3,55.1$, 
65.0, 78.8, 81.9, 82.3, 106.1, 127.8, 127.8, 129.8, 129.8, 133.5, 133.6, 135.7, 135.8, 140.3, 143.0, 159.8, 161.6. IR (thin film): 702, 838, 1112, 1255, 1275, 1713, 2858, 2931, $2959 \mathrm{~cm}^{-1}$. HRMS (ESI) calc. for $\mathrm{C}_{39} \mathrm{H}_{59} \mathrm{O}_{7} \mathrm{Si}_{2}:(\mathrm{M}+\mathrm{H})^{+}, 695.3800$, found: $(\mathrm{M}+\mathrm{H})^{+}, 695.3799$.

\section{Lactone 12}

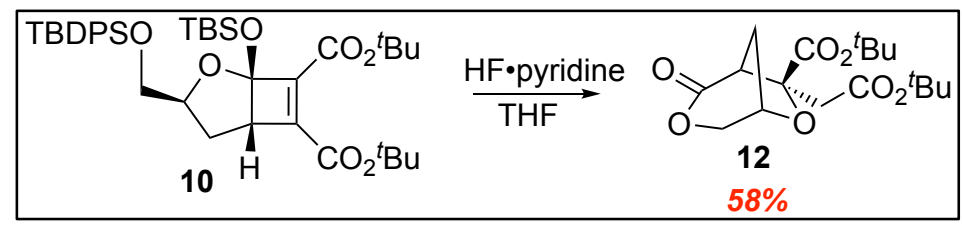

To a solution of cyclobutene $\mathbf{1 0}(3 \mathrm{~g}, 4.32 \mathrm{mmol})$ in THF $(25 \mathrm{~mL})$ was added HF•pyridine $(70 \%, 1.1$ $\mathrm{mL}, 43.2 \mathrm{mmol})$ and the resultant solution stirred at $\mathrm{rt}$ for 24 hours. The mixture was diluted with EtOAc $(25 \mathrm{~mL})$, quenched with solid $\mathrm{NaHCO}_{3}(5 \mathrm{~g})$ and the result suspension was filtered through a pad of celite and the filter cake was washed with EtOAc. The solvent was concentrated under reduced pressure. Purification by flash chromatography with 50\% EtOAc/Petrol as eluent afforded lactone 12 (857 mg, 58\%) as a yellow solid. m.p. $107.3-111.0^{\circ} \mathrm{C} .[\alpha]_{\mathrm{D}}=+39.5\left(c 1.15, \mathrm{CHCl}_{3}\right) .{ }^{1} \mathrm{H}$ NMR $(600$ $\left.\mathrm{MHz} \mathrm{CDCl}_{3}\right) \delta 1.44(\mathrm{~s}, 9 \mathrm{H}), 1.47(\mathrm{~s}, 9 \mathrm{H}), 2.10(\mathrm{~d}, J=12.2,1 \mathrm{H}), 2.54(\mathrm{dddd}, J=12.1,5.9,4.2,1.8$ $\mathrm{Hz}, 1 \mathrm{H}), 2.69$ (d, $J=17.2 \mathrm{~Hz}, 2 \mathrm{H}), 3.01$ (d, $J=17.2 \mathrm{~Hz}, 1 \mathrm{H}), 3.31$ (dt, $J=4.0,0.8 \mathrm{~Hz}, 1 \mathrm{H}), 4.20$ (dd, $J=11.7,1.8 \mathrm{~Hz}, 1 \mathrm{H}), 4.33(\mathrm{dt}, J=11.7,1.0 \mathrm{~Hz}, 2 \mathrm{H}), 4.67$ (br d, $J=5.9 \mathrm{~Hz}, 1 \mathrm{H}) .{ }^{13} \mathrm{C}$ NMR $(101$ $\left.\mathrm{MHz}_{,} \mathrm{CDCl}_{3}\right) \delta 27.9,28.2,28.8,29.8,41.6,49.2,74.0,75.6,81.7,82.6,84.3,168.6,168.9,169.6$. IR (film): 1058, 1094, 1148, 1223, 1363, 1725, 1751, 2953, $2981 \mathrm{~cm}^{-1}$. HRMS (ESI): calc. for $\mathrm{C}_{17} \mathrm{H}_{26} \mathrm{O}_{7} \mathrm{Na}:(\mathrm{M}+\mathrm{Na})^{+}, 365.1577$, found: $(\mathrm{M}+\mathrm{Na})^{+}, 365.1572$.

\section{Alcohol 11}

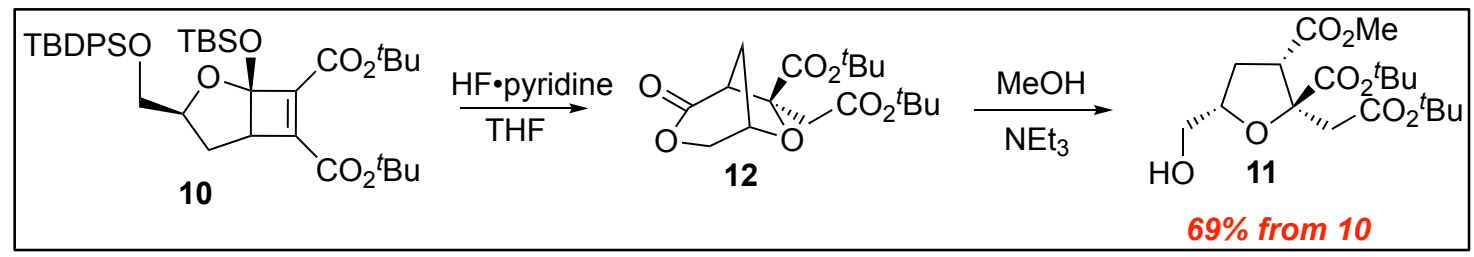

To a solution of cyclobutene $\mathbf{1 0}(3 \mathrm{~g}, 4.3 \mathrm{mmol})$ in THF (25 mL) was added HF•pyridine (70\%, 1.1 $\mathrm{mL}, 43.2 \mathrm{mmol})$ and the resultant solution stirred at r.t. for 24 hours. The mixture was diluted with EtOAc $(25 \mathrm{~mL})$, quenched with solid $\mathrm{NaHCO}_{3}(5 \mathrm{~g})$ and the resultant suspension was filtered through a pad of Celite and the fliter cake was washed with EtOAc. The solvent was concentrated under reduced pressure. The crude lactone 12 was dissolved in $\mathrm{MeOH}(25 \mathrm{~mL})$ and $\mathrm{NEt}_{3}(3.0 \mathrm{~mL}, 21.6$ mmol) was added and the resultant solution stirred at rt. for $16 \mathrm{~h}$. Concentration of the reation mixture under reduced pressure and purification of the crude product by flash chromatography $(50 \%$ EtOAc/Petrol) afforded alcohol $11(1.11 \mathrm{~g}, 69 \%$, over 2 steps $)$ as a yellow oil. $[\alpha]_{\mathrm{D}}=+47.1(c 1.12$, $\mathrm{CH}_{2} \mathrm{Cl}_{2}$ ). ${ }^{1} \mathrm{H}$ NMR $\left(500 \mathrm{MHz}, \mathrm{CDCl}_{3}\right) \delta 1.42(\mathrm{~s}, 9 \mathrm{H}), 1.48(\mathrm{~s}, 9 \mathrm{H}), 2.19$ (ddd, $J=12.7,7.6,7.0 \mathrm{~Hz}$, $1 \mathrm{H}), 2.43(\mathrm{dt}, J=12.7,9.2 \mathrm{~Hz}, 1 \mathrm{H}), 2.80(\mathrm{ABq}, J=15.0 \mathrm{~Hz}, 2 \mathrm{H}), 3.36(\mathrm{dd}, J=9.1,7.9 \mathrm{~Hz}, 1 \mathrm{H}), 3.53$ $(\mathrm{dd}, J=12.4,3.1 \mathrm{~Hz}, 1 \mathrm{H}), 3.70(\mathrm{~s}, 3 \mathrm{H}), 3.90(\mathrm{dd}, J=12.4,2.6 \mathrm{~Hz}, 1 \mathrm{H}), 4.33(\mathrm{ddt}, J=6.3,6.0,3.0$ $\mathrm{Hz}, 1 \mathrm{H}) .{ }^{13} \mathrm{C} \mathrm{NMR}\left(126 \mathrm{MHz}, \mathrm{CDCl}_{3}\right) \delta 28.0,28.1,30.0,41.1,50.8,52.2,63.0,80.7,81.8,82.3$, 84.7, 169.7, 170.3, 172.2. IR (film): 845, 1060, 1147, 1367, 1730, 2934, 2979, $3466 \mathrm{~cm}^{-1}$. HRMS (ESI) calc. for $\mathrm{C}_{18} \mathrm{H}_{30} \mathrm{O}_{8} \mathrm{Na}:(\mathrm{M}+\mathrm{Na})^{+}, 397.1839$, found: $(\mathrm{M}+\mathrm{Na})^{+}, 397.1835$. 


\section{Tosylate}

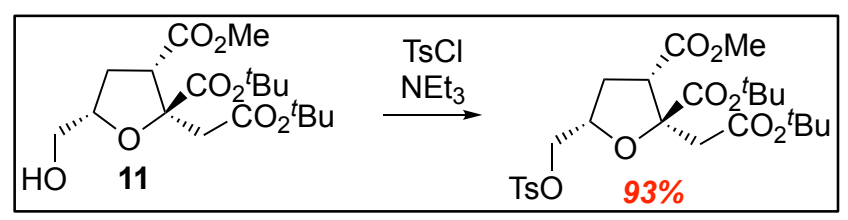

To a solution of alcohol $11(2 \mathrm{~g}, 5.34 \mathrm{mmol})$ in dry $\mathrm{CH}_{2} \mathrm{Cl}_{2}(26 \mathrm{~mL})$ at $0^{\circ} \mathrm{C}$ was added $\mathrm{TsCl}(1.52 \mathrm{~g}$, $8.01 \mathrm{mmol}), \mathrm{NEt}_{3}(2.2 \mathrm{~mL}, 16.02 \mathrm{mmol})$ and DMAP $(65 \mathrm{mg}, 0.53 \mathrm{mmol})$ and after stirring for 30 $\mathrm{min}$, the reaction was warmed to $\mathrm{rt}$ over $2 \mathrm{~h}$. The resulting deep purple solution was diluted with EtOAc and $\mathrm{H}_{2} \mathrm{O}$ and the aqueous phase was extracted with EtOAc. The combined organic layers were washed with $1 \mathrm{M}$ aqueous $\mathrm{HCl}$, sat.aqueous $\mathrm{NaHCO}_{3}, \mathrm{H}_{2} \mathrm{O}$ and brine then dried with $\mathrm{Na}_{2} \mathrm{SO}_{4}$ and concentrated under reduced pressure. The crude product was purified by flash chromatography with $20 \% \mathrm{EtOAc} /$ petrol as eluent to give tosylate $(2.62 \mathrm{~g}, 93 \%)$ as a yellow oil. $[\alpha]_{\mathrm{D}}=+35.7$ (c 1.2, $\left.\mathrm{CH}_{2} \mathrm{Cl}_{2}\right) .{ }^{1} \mathrm{H}$ NMR $\left(500 \mathrm{MHz}, \mathrm{CDCl}_{3}\right) \delta 1.40(\mathrm{~s}, 9 \mathrm{H}), 1.45(\mathrm{~s}, 9 \mathrm{H}), 2.09(\mathrm{dt}, J=13.2,6.6 \mathrm{~Hz}, 1 \mathrm{H})$, $2.31(\mathrm{dt}, J=13.1,7.8 \mathrm{~Hz}, 1 \mathrm{H}), 2.43(\mathrm{~s}, 3 \mathrm{H}), 2.68(\mathrm{ABq}, J=16.9 \mathrm{~Hz}, 2 \mathrm{H}), 3.36(\mathrm{dd}, J=8.2,6.4 \mathrm{~Hz}$, $1 \mathrm{H}), 3.67(\mathrm{~s}, 3 \mathrm{H}), 4.13(\mathrm{~d}, J=5.5 \mathrm{~Hz}, 2 \mathrm{H}), 4.39(\mathrm{~m}, 1 \mathrm{H}), 7.32(\mathrm{~d}, J=8.3 \mathrm{~Hz}, 2 \mathrm{H}), 7.78(\mathrm{~d}, J=8.3$ $\mathrm{Hz}, 2 \mathrm{H}) .{ }^{13} \mathrm{C} \mathrm{NMR}\left(126 \mathrm{MHz}, \mathrm{CDCl}_{3}\right) \delta 21.8,27.9,28.1,31.8,40.4,49.8,52.2,70.7,76.8,81.3$, $82.3,85.7,128.2,129.9,132.9,144.9,168.8,170.4,172.2$. IR (thin film): 664, 814, 836, 958, 984, 1146, 1174, 1365, 1732, $2979 \mathrm{~cm}^{-1}$. HRMS (ESI) calc. for $\mathrm{C}_{25} \mathrm{H}_{36} \mathrm{O}_{10} \mathrm{SNa}:(\mathrm{M}+\mathrm{Na})^{+}, 551.1927$, found: $(\mathrm{M}+\mathrm{Na})^{+}, 551.1924$.

\section{Alkene 13}

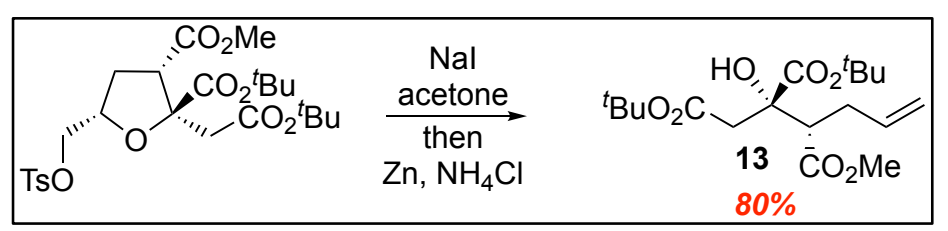

A solution of the tosylate $(2.51 \mathrm{~g}, 4.7 \mathrm{mmol})$ and sodium iodide $(11.98 \mathrm{~g}, 47.3 \mathrm{mmol})$ in acetone (30 $\mathrm{mL}$ ) was heated to reflux for $12 \mathrm{~h}$ then cooled and filtered through a pad of celite. The filtrate was concentrated and the residue was dissolved in $\mathrm{EtOH}(95 \%, 50 \mathrm{~mL})$ and activated zinc power $(3.09 \mathrm{~g}$, $47.3 \mathrm{mmol})$ and $\mathrm{NH}_{4} \mathrm{Cl}(1.26 \mathrm{~g}, 23.6 \mathrm{mmol})$ were added and the mixture was stirred at $\mathrm{rt}$ for $3 \mathrm{~h}$. The mixture was diluted with $\mathrm{Et}_{2} \mathrm{O}(50 \mathrm{~mL})$, filtered through a pad of celite. The filtrate was concentrated and the residue was purified by flash chromatography with $10 \% \mathrm{EtOAc} /$ petrol as eluent to afford the alkene $13(2.02 \mathrm{~g}, 80 \%)$ as a clear oil. $[\alpha]_{\mathrm{D}}=+10.6\left(c\right.$ 0.67, $\left.\mathrm{CH}_{2} \mathrm{Cl}_{2}\right) .{ }^{1} \mathrm{H} \mathrm{NMR}\left(500 \mathrm{MHz}, \mathrm{CDCl}_{3}\right) \delta$ $1.42(\mathrm{~s}, 9 \mathrm{H}), 1.49(\mathrm{~s}, 9 \mathrm{H}), 2.30(\mathrm{~m}, 1 \mathrm{H}), 2.51(\mathrm{~m}, 1 \mathrm{H}), 2.62(\mathrm{~d}, J=16.7 \mathrm{~Hz}, 1 \mathrm{H}) 2.73(\mathrm{dd}, J=11.9$, $3.1 \mathrm{~Hz}, 1 \mathrm{H}), 2.98(\mathrm{~d}, J=16.7 \mathrm{~Hz}, 2 \mathrm{H}), 3.69(\mathrm{~s}, 3 \mathrm{H}), 3.96(\mathrm{~s}, 1 \mathrm{H}), 5.00(\mathrm{~d}, J=9.8 \mathrm{~Hz}, 1 \mathrm{H}), 5.05(\mathrm{dd}$, $J=17.1 \mathrm{~Hz}, 1 \mathrm{H}), 5.67(\mathrm{~m}, 1 \mathrm{H}) .{ }^{13} \mathrm{C} \mathrm{NMR}\left(126 \mathrm{MHz}, \mathrm{CDCl}_{3}\right)$ 27.9, 28.2, 31.6, 41.8, 51.9, 53.7, 75.2, 81.6, 83.4, 117.3, 135.0, 170.0, 172.2, 172.8. IR (film): 8451 1149, 1249, 1368, 1730, 2935, 2980, $3484 \mathrm{~cm}^{-1}$. HRMS (ESI) calc. for $\mathrm{C}_{18} \mathrm{H}_{30} \mathrm{O}_{7} \mathrm{Na}:(\mathrm{M}+\mathrm{Na})^{+}, 381.1890$, found: $(\mathrm{M}+\mathrm{Na})^{+}, 381.1887$.

\section{Silyl ether 14}

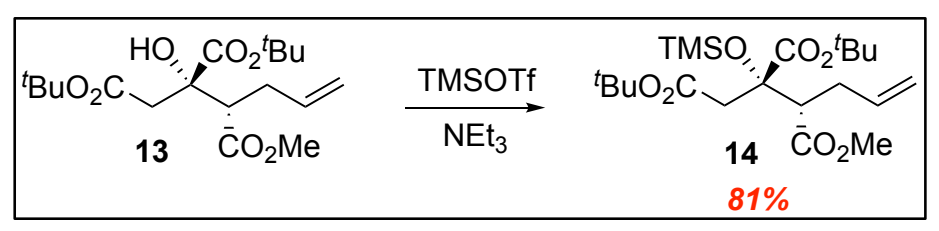


To a solution of $\mathrm{Et}_{3} \mathrm{~N}(300 \mu \mathrm{l}, 2.1 \mathrm{mmol})$ and triester $13(500 \mathrm{mg}, 1.4 \mathrm{mmol})$ in anhydrous $\mathrm{CH}_{2} \mathrm{Cl}_{2}$ $(10 \mathrm{ml})$ at $0{ }^{\circ} \mathrm{C}$ was added TMSOTf $(370,1.7 \mathrm{mmol})$ and the solution was stirred for $2 \mathrm{~h}$. The mixture was warmed to $\mathrm{rt}$ and stirred for $1 \mathrm{~h}$ the cooled to $0{ }^{\circ} \mathrm{C}$, diluted with petroleum ether and quenched with $\mathrm{H}_{2} \mathrm{O}$. The aqueous phase was extracted with petroleum ether and the combined organic layers were washed with brine then dried over $\mathrm{Na}_{2} \mathrm{SO}_{4}$ and concentrated. Purification by flash chromatography with 5\% EtOAc/petrol as eluent afforded silyl ether 14 (488 $\mathrm{mg}, 81 \%)$ as a clear oil. $[\alpha]_{\mathrm{D}}=+5.6\left(c\right.$ 2.0, $\left.\mathrm{CH}_{2} \mathrm{Cl}_{2}\right) .{ }^{1} \mathrm{H}$ NMR $\left(600 \mathrm{MHz}, \mathrm{CDCl}_{3}\right) \delta 0.16(\mathrm{~s}, 9 \mathrm{H}), 1.43(\mathrm{~s}, 9 \mathrm{H}), 1.46(\mathrm{~s}, 9 \mathrm{H})$, $2.50-2.37(\mathrm{~m}, 2 \mathrm{H}), 2.62(\mathrm{~d}, J=16.0 \mathrm{~Hz}, 1 \mathrm{H}), 2.78(\mathrm{dd}, J=11.5,3.4 \mathrm{~Hz}, 1 \mathrm{H}), 2.96(\mathrm{~d}, J=16.0 \mathrm{~Hz}$, $1 \mathrm{H}), 3.65(\mathrm{~s}, 3 \mathrm{H}), 5.00(\mathrm{dd}, J=10.1,0.7 \mathrm{~Hz}, 1 \mathrm{H}), 5.05(\mathrm{dq}, J=17.6,1.5 \mathrm{~Hz}, 2 \mathrm{H}), 5.68$ (dddd, $J=$ 17.0, 10.1, 7.1, 6.6, 1H). ${ }^{13} \mathrm{C}$ NMR $\left(151 \mathrm{MHz}, \mathrm{CDCl}_{3}\right) \delta$ 2.6, 28.0, 28.3, 32.2, 42.7, 51.6, 54.8, 78.7, 80.6, 82.2, 117.0, 135.4, 169.1, 171.5, 172.5. IR (film): 840, 1145, 1247, 1368, 1738, 2954, $2980 \mathrm{~cm}^{-}$ 1. HRMS (ESI) calc. for $\mathrm{C}_{21} \mathrm{H}_{38} \mathrm{O}_{7} \mathrm{SiNa}:(\mathrm{M}+\mathrm{Na})^{+}, 453.2285$, found: $(\mathrm{M}+\mathrm{Na})^{+}, 453.2278$.

\section{Alkyne 8 (Procedure reported by Barrett et al.) ${ }^{1}$}

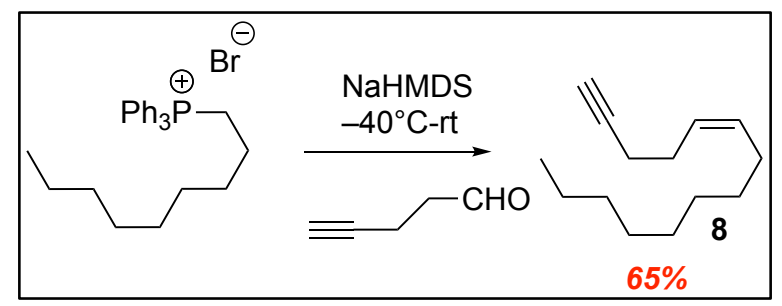

To a solution of (1-nonyl)triphenylphosphonium bromide (5.4 g, $11.5 \mathrm{mmol})$ in anhydrous THF (50 $\mathrm{ml}$ ) was added LiHMDS in THF $(1 \mathrm{M} ; 10.6 \mathrm{~mL}, 10.6 \mathrm{mmol})$ dropwise at $-40^{\circ} \mathrm{C}$. The resulting redorange solution was warmed to $0^{\circ} \mathrm{C}$ for $1 \mathrm{~h}$ and cooled back to $-40{ }^{\circ} \mathrm{C}$. 4-Pentynal $(788 \mathrm{mg}, 9.6 \mathrm{mmol})$ in THF $(5 \mathrm{~mL})$ was added dropwise and the mixture was warmed to $0{ }^{\circ} \mathrm{C}$ for $3 \mathrm{~h}$. The resulting yellow solution was diluted with $\mathrm{Et}_{2} \mathrm{O}(50 \mathrm{~mL})$ and quenched with sat. $\mathrm{NH}_{4} \mathrm{Cl}$. The organic layer was washed with brine, dried $\mathrm{MgSO}_{4}$ and concentrated. Purification of the residue by flash chromatography with petrol as eluent afforded alkyne $8(1.2 \mathrm{~g}, 65 \%)$ as a colourless oil. ${ }^{1} \mathrm{H} \mathrm{NMR}\left(600 \mathrm{MHz}, \mathrm{CDCl}_{3}\right) \delta 0.87$ $(\mathrm{t}, J=6.9,3 \mathrm{H}), 1.38-1.20(\mathrm{~m}, 12 \mathrm{H}), 1.94(\mathrm{t}, J=2.4 \mathrm{~Hz}, 1 \mathrm{H}), 2.04(\mathrm{q}, J=7.2 \mathrm{~Hz}, 2 \mathrm{H}), 2.31-2.19$ (m, 4H), 5.37-5.47 (m, 2H). ${ }^{13} \mathrm{C}$ NMR (151 MHz, $\left.\mathrm{CDCl}_{3}\right) \delta 14.3,19.0,22.8,26.5,27.5,29.5,29.7$, 29.8, 32.0, 68.4, 84.4, 127.5, 131.9. IR (film): 722, 1465, 2854, 2924, 2956, $3314 \mathrm{~cm}^{-1}$.

\section{Allylic alcohol 16}

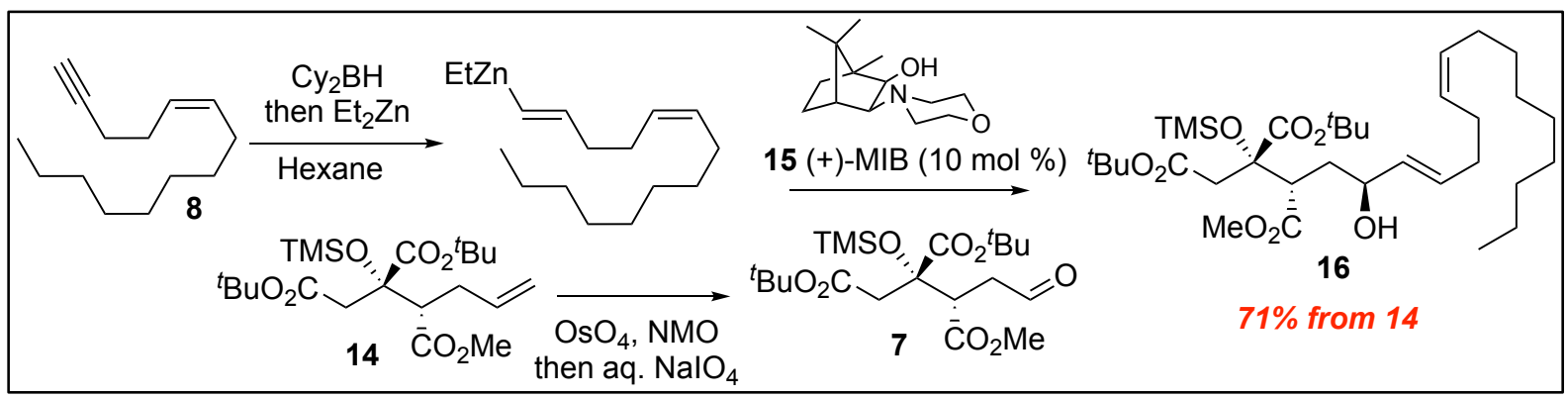

To a solution of NMO (140 mg, $1.15 \mathrm{mmol})$ and alkene $14(100 \mathrm{mg}, 0.23 \mathrm{mmol})$ in $\mathrm{THF} / \mathrm{H}_{2} \mathrm{O}(3: 1,4$ $\mathrm{ml})$ was added $\mathrm{OsO}_{4}(59 \mu 1,0.39 \mathrm{M}$ in benzene, $0.023 \mathrm{mmol})$ and the resultant mixture was stirred for 4h. $\mathrm{NaIO}_{4}(197 \mathrm{mg}, 0.92 \mathrm{mmol})$ was then added and the mixture was stirred for a further $16 \mathrm{~h}$. The reaction mixture was diluted with $\mathrm{Et}_{2} \mathrm{O}$ and quenched with sat. aqueous $\mathrm{Na}_{2} \mathrm{~S}_{2} \mathrm{O}_{3}$. The aqueous phase was extracted with $\mathrm{Et}_{2} \mathrm{O}$ and the combined organic layers were washed with $\mathrm{H}_{2} \mathrm{O}$, brine then dried 
over $\mathrm{Na}_{2} \mathrm{SO}_{4}$ and concentrated. The crude aldehyde 7 (99.4 mg, $\left.0.23 \mathrm{mmol}, 100 \%\right)$ was used directly next reaction without further purification.

To a suspension of freshly prepared $\mathrm{Cy}_{2} \mathrm{BH}(110 \mathrm{mg}, 0.62 \mathrm{mmol})$ in anhydrous hexane $(1 \mathrm{ml})$ at $0{ }^{\circ} \mathrm{C}$ was added a solution of alkyne $8(133 \mathrm{mg}, 0.69 \mathrm{mmol})$ in hexane $(1 \mathrm{ml})$ dropwise. The suspension was warmed to rt and stirred for $1 \mathrm{~h}$ until a clear solution was obtained. This solution was cooled to $78{ }^{\circ} \mathrm{C}$ and a solution of $\mathrm{ZnEt}_{2}(1 \mathrm{M}$ in hexanes, $620 \mu \mathrm{L}, 0.62 \mathrm{mmol})$ was added followed by and a solution of $(+)-\mathrm{MIB}^{2}(11 \mathrm{mg}, 0.046 \mathrm{mmol})$ in hexane $(1 \mathrm{ml})$ dropwise. The reaction mixture was warmed to $-20^{\circ} \mathrm{C}$ for $1 \mathrm{~h}$ to form a grey suspension and a solution of crude aldehyde in hexane $(1 \mathrm{ml})$ was added dropwise. The suspension was stirred under $0{ }^{\circ} \mathrm{C}$ for $2 \mathrm{~h}$ and quenched with $\mathrm{H}_{2} \mathrm{O}$. The aqueous phase was extracted with EtOAc and the combined organic layers were washed with brine then dried over $\mathrm{Na}_{2} \mathrm{SO}_{4}$ and concentrated. Purification by flash chromatography with $10 \%$ EtOAc/petrol as eluent afforded allylic alcohol $16(102 \mathrm{mg}, 71 \%$ over 2 steps $)$ as a clear oil. $[\alpha]_{\mathrm{D}}=$ 3.6 (c 1.3, $\left.\mathrm{CH}_{2} \mathrm{Cl}_{2}\right) .{ }^{1} \mathrm{H}$ NMR $\left(500 \mathrm{MHz}, \mathrm{CDCl}_{3}\right) \delta 0.16(\mathrm{~s}, 9 \mathrm{H}) 0.88(\mathrm{t}, J=6.8 \mathrm{~Hz}, 3 \mathrm{H}), 1.22-133(\mathrm{~m}$, $12 \mathrm{H}), 1.43(\mathrm{~s}, 9 \mathrm{H}), 1.47(\mathrm{~s}, 9 \mathrm{H}), 1.61(\mathrm{~m}, 1 \mathrm{H}), 1.81(\mathrm{ddd}, J=13.8,8.6,2.2 \mathrm{~Hz}, 1 \mathrm{H}), 1.98-2.11(\mathrm{~m}$, $6 \mathrm{H}), 2.60(\mathrm{~d}, J=16.0 \mathrm{~Hz}, 1 \mathrm{H}), 2.93(\mathrm{~d}, J=16.0 \mathrm{~Hz}, 1 \mathrm{H}), 3.03(\mathrm{dd}, J=11.6,1.8 \mathrm{~Hz}, 1 \mathrm{H}), 3.68(\mathrm{~s}$, $3 \mathrm{H}), 3.98$ (br s, 1H), $5.32-5.39(\mathrm{~m}, 2 \mathrm{H}), 5.47$ (dd, $J=15.4,6.8 \mathrm{~Hz}, 1 \mathrm{H}), 5.66$ (dt, $J=15.4,6.1 \mathrm{~Hz}$, 1H). ${ }^{13} \mathrm{C} \mathrm{NMR}\left(151 \mathrm{MHz}, \mathrm{CDCl}_{3}\right) \delta 2.6,14.3,22.81,22.83,27.0,27.4,28.0,28.3,29.5,29.5,29.7$, 29.9, 31.7, 32.0, 32.5, 35.6, 42.8, 50.9, 51.8, 71.0, 78.8, 80.7, 82.2, 128.8, 130.8, 131.8, 132.7, 169.3, 171.5, 173.3. IR (film): 844, 1151, 1247, 1368, 1740, 2856, 2927, 2955, $3491 \mathrm{~cm}^{-1}$. HRMS (ESI) calc. for $\mathrm{C}_{34} \mathrm{H}_{62} \mathrm{O}_{8} \mathrm{SiNa}:(\mathrm{M}+\mathrm{Na})^{+}, 649.4112$, found: $(\mathrm{M}+\mathrm{Na})^{+}, 649.4109$.

\section{Lactone 17}

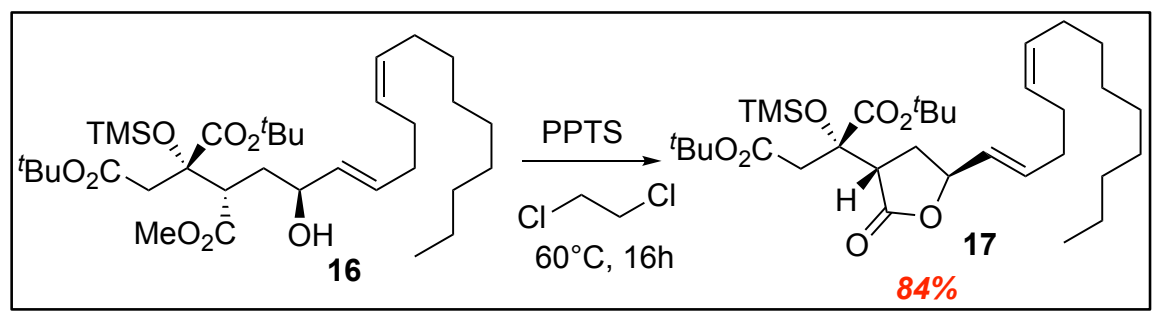

To a solution of the alcohol $16(80 \mathrm{mg}, 0.13 \mathrm{mmol})$ in 1,2-dichloroethane $(10 \mathrm{~mL})$ at $0^{\circ} \mathrm{C}$ was added PPTS $(6.4 \mathrm{mg}, 0.025 \mathrm{mmol})$. The solution was stirred at $60^{\circ} \mathrm{C}$ for $16 \mathrm{~h}$ and then concentrated. Purification by flash chromatography with 5\% EtOAc/petrol as eluent afforded the lactone 17 (65 mg, 84\%) as clear oil. $[\alpha]_{\mathrm{D}}=-11.6\left(c\right.$ 1.5, $\left.\mathrm{CH}_{2} \mathrm{Cl}_{2}\right) .{ }^{1} \mathrm{H} \mathrm{NMR}\left(500 \mathrm{MHz}, \mathrm{CDCl}_{3}\right) \delta 0.17(\mathrm{~s}, 9 \mathrm{H}), 0.87$ $(\mathrm{t}, J=6.9 \mathrm{~Hz}, 3 \mathrm{H}), 1.27-1.33(\mathrm{~m}, 12 \mathrm{H}), 1.45(\mathrm{~s}, 9 \mathrm{H}), 1.47(\mathrm{~s}, 9 \mathrm{H}), 1.99-2.03(\mathrm{~m}, 3 \mathrm{H}), 2.10-2.13(\mathrm{~m}$, 4H), $2.48(\mathrm{ddd}, J=13.2,7.7,5.4 \mathrm{~Hz}, 1 \mathrm{H}), 3.10(\mathrm{dd}, J=10.0,5.2 \mathrm{~Hz}, 1 \mathrm{H}), 3.15(\mathrm{~s}, 3 \mathrm{H}), 4.88(\mathrm{q}, J=$ $7.2 \mathrm{~Hz}, 1 \mathrm{H}), 5.30-5.42(\mathrm{~m}, 2 \mathrm{H}), 5.45(\mathrm{dd}, J=15.3,7.3 \mathrm{~Hz}, 1 \mathrm{H}), 5.76(\mathrm{~m}, 1 \mathrm{H}) .{ }^{13} \mathrm{C} \mathrm{NMR}(126 \mathrm{MHz}$, $\left.\mathrm{CDCl}_{3}\right) \delta 2.3,14.3,22.8,26.6,27.4,28.0,28.2,29.45,29.46,29.6,29.8,31.3,32.0,32.3,43.0,48.1$, 79.0, 79.8, 80.9, 82.2, 128.4, 128.7, 131.0, 134.7, 169.3, 170.7, 175.9. IR (thin film): 756, 842, 1106, 1149, 1249, 1368, 1734, 1748, 1770, 2856, 2926, $2956 \mathrm{~cm}^{-1}$. HRMS (ESI) calc. for $\mathrm{C}_{33} \mathrm{H}_{58} \mathrm{O}_{7} \mathrm{SiNa}$ : $(\mathrm{M}+\mathrm{Na})^{+}, 617.3850$, found: $(\mathrm{M}+\mathrm{Na})^{+}, 617.3845$.

Vinyl iodide 20 (alternative proceedure to that reported) ${ }^{3}$

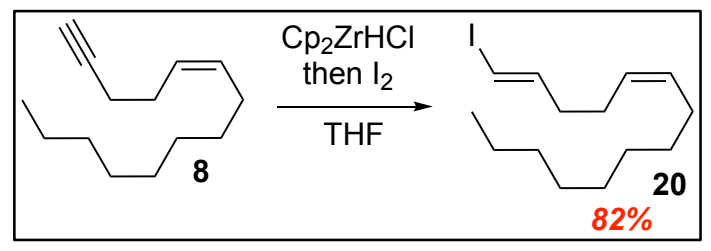


A solution of alkyne $4(400 \mathrm{mg}, 2.1 \mathrm{mmol})$ in THF $(10 \mathrm{ml})$ in a foil wrapped flask was treated with Schwartz's reagent $(567 \mathrm{mg}, 2.2 \mathrm{mmol})$ at $-20{ }^{\circ} \mathrm{C}$ and stirred in the dark for $1 \mathrm{~h}$. The reaction mixture was added a solution of $\mathrm{I}_{2}(304 \mathrm{mg}, 1.2 \mathrm{mmol})$ in THF $(3 \mathrm{~mL})$ and stirred for a further $30 \mathrm{~min}$. Sat. aqueous $\mathrm{Na}_{2} \mathrm{~S}_{2} \mathrm{O}_{3}$ was then added to quench reaction. The aqueous phase was extracted with hexane and the combined organic layers were washed with brine then dried over $\mathrm{Na}_{2} \mathrm{SO}_{4}$ and concentrated. Purification by flash chromatography with $100 \%$ petrol as eluent afforded vinyl iodide $20(551 \mathrm{mg}, 82 \%)$ as a yellow oil. ${ }^{1} \mathrm{H}$ NMR $\left(600 \mathrm{MHz}, \mathrm{CDCl}_{3}\right) \delta 0.88(\mathrm{t}, J=7.0 \mathrm{~Hz}, 3 \mathrm{H})$, $1.26-1.30(\mathrm{~m}, 12 \mathrm{H}), 2.00(\mathrm{q}, J=7.0 \mathrm{~Hz}, 2 \mathrm{H}), 2.15-2.07(\mathrm{~m}, 4 \mathrm{H}), 5.31(\mathrm{~m}, 1 \mathrm{H}), 5.41(\mathrm{~m}, 1 \mathrm{H}), 6.01$ $(\mathrm{dt}, J=14.3,1.3 \mathrm{~Hz}, 1 \mathrm{H}), 6.52(\mathrm{dt}, J=14.3,7.0 \mathrm{~Hz}, 1 \mathrm{H}) .{ }^{13} \mathrm{C} \mathrm{NMR}\left(151 \mathrm{MHz}, \mathrm{CDCl}_{3}\right): \delta 14.3,22.8$, 26.3, 27.4, 29.5, 29.5, 29.7, 29.8, 32.1, 36.2, 74.9, 127.9, 131.4, 146.2.

\section{Kishi-Nozaki coupling: Undesired cis-Lactone 21}

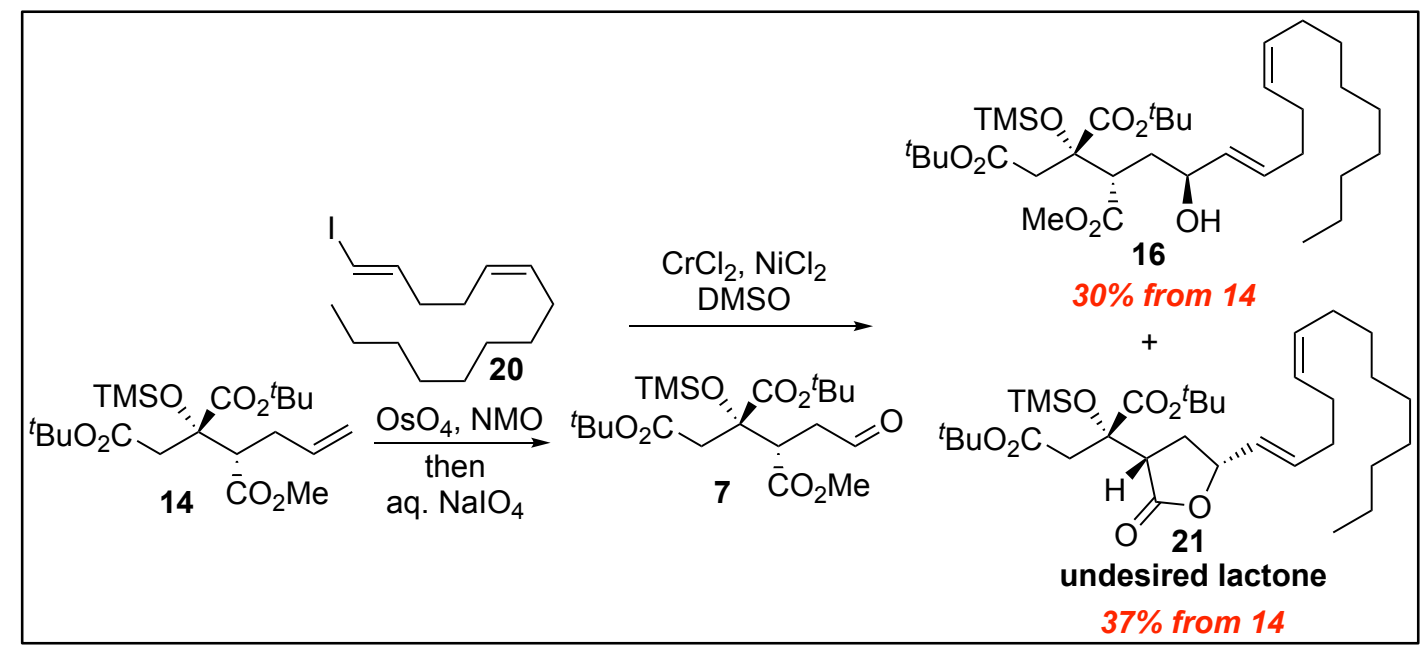

A solution of NMO (162 mg, $1.38 \mathrm{mmol})$ and silyl ester $14(119 \mathrm{mg}, 0.28 \mathrm{mmol})$ in $\mathrm{THF} / \mathrm{H}_{2} \mathrm{O}(3: 1$, $4 \mathrm{ml})$ was added $\mathrm{OsO}_{4}(71 \mu \mathrm{l}, 0.39 \mathrm{M}$ in benzene, $0.028 \mathrm{mmol})$ and stirred for $4 \mathrm{~h}$. The resulting solution was added $\mathrm{NaIO}_{4}(238 \mathrm{mg}, 1.11 \mathrm{mmol})$ and kept stirred further $16 \mathrm{~h}$. The reaction mixture was quenched by $\mathrm{Na}_{2} \mathrm{~S}_{2} \mathrm{O}_{3}$ (sat.). The aqueous phase was extracted with EtOAc and the combined organic layers were washed with $\mathrm{H}_{2} \mathrm{O}$, brine then dried over $\mathrm{Na}_{2} \mathrm{SO}_{4}$ and concentrated to give the crude aldehyde which was used directly next reaction without further purification. A mixture of $\mathrm{NiCl}_{2}$ (3.6 mg, $0.028 \mathrm{mmol}$ ) and $\mathrm{CrCl}_{2}(206 \mathrm{mg}, 1.68 \mathrm{mmol})$ in anhydrous DMSO (degassed, $3 \mathrm{ml}$ ) was stirred for $30 \mathrm{~min}$. A mixture of aldehyde $(120 \mathrm{mg}, 0.28 \mathrm{mmol})$ and vinyl iodide 3 (445 $\mathrm{mg}, 1.39$ $\mathrm{mmol}$ ) in anhydrous DMSO (degassed, $3 \mathrm{ml}$ ) were added to the mixture at $\mathrm{rt}$ and the resulting mixture was stirred at $50{ }^{\circ} \mathrm{C}$ for $24 \mathrm{~h}$. The reaction mixture was diluted with water and extracted with $\mathrm{Et}_{2} \mathrm{O}$, washed with brine, dried, concentrated, and chromatographed with 5\% EtOAc/petrol as eluent to give the undesired lactone 21 (49 mg, 30\%). [ $\alpha]_{\mathrm{D}}=-36.6\left(c 0.76, \mathrm{CH}_{2} \mathrm{Cl}_{2}\right) .{ }^{1} \mathrm{H} \mathrm{NMR}\left(600 \mathrm{MHz}, \mathrm{CDCl}_{3}\right)$ $\delta 0.16(\mathrm{~s}, 9 \mathrm{H}), 0.88(\mathrm{t}, J=7.0 \mathrm{~Hz}, 3 \mathrm{H}), 1.35-1.22(\mathrm{~m}, 12 \mathrm{H}), 1.44(\mathrm{~s}, 9 \mathrm{H}), 1.46(\mathrm{~s}, 9 \mathrm{H}), 2.00(\mathrm{q}, J=$ $7.0 \mathrm{~Hz}, 2 \mathrm{H}), 2.12-2.19(\mathrm{~m}, 5 \mathrm{H}), 2.29(\mathrm{ddd}, J=12.7,8.8,6.4 \mathrm{~Hz}, 1 \mathrm{H}), 3.21(\mathrm{dd}, J=11.5,8.9 \mathrm{~Hz}$, $1 \mathrm{H}), 3.25(\mathrm{ABq}, J=16.6 \mathrm{~Hz}, 2 \mathrm{H}), 4.67(\mathrm{dt}, J=10.2,7.0 \mathrm{~Hz}, 1 \mathrm{H}), 5.33(\mathrm{~m}, 1 \mathrm{H}), 5.39(\mathrm{~m}, 1 \mathrm{H}), 5.47$ $(\mathrm{dd}, J=15.3,7.6 \mathrm{~Hz}, 1 \mathrm{H}), 5.80(\mathrm{~m}, 1 \mathrm{H}) .{ }^{13} \mathrm{C} \mathrm{NMR}\left(151 \mathrm{MHz}, \mathrm{CDCl}_{3}\right) \delta 2.4,14.3,22.8,26.7,27.4$, 28.0, 28.3, 29.4, 29.5, 29.7, 29.8, 31.3, 32.0, 32.4, 42.5, 48.5, 77.6, 78.5, 80.9, 82.2, 128.2, 128.4, 131.0, 135.6, 169.5, 170.9, 175.0. IR (thin firm): 842, 1150, 1248, 1368, 1733, 1749, 1772, 2855, 2926, $2956 \mathrm{~cm}^{-1}$. HRMS (ESI) calc. for $\mathrm{C}_{33} \mathrm{H}_{58} \mathrm{O}_{7} \mathrm{SiNa}:(\mathrm{M}+\mathrm{Na})^{+}, 617.3850$, found: $(\mathrm{M}+\mathrm{Na})^{+}$, 617.3846.

Further elution with 10\% EtOAc/Petrol gave the desired alcohol 16 (65 mg, 37\%). 


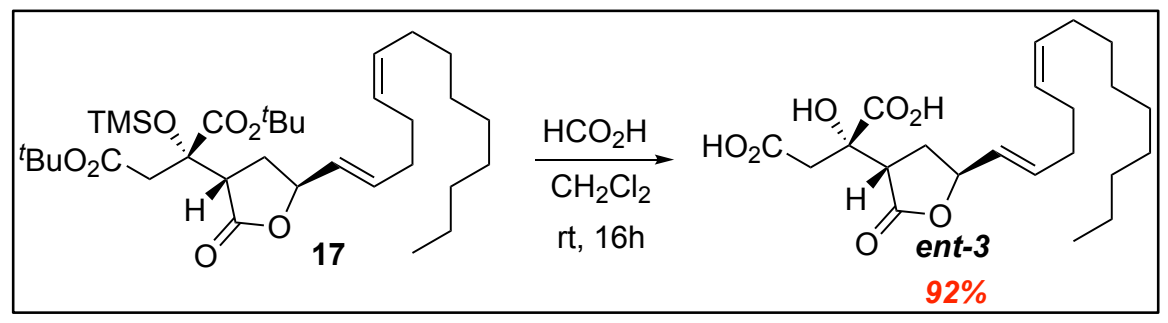

To a solution of the lactone $17(20 \mathrm{mg}, 0.034 \mathrm{mmol})$ in $\mathrm{CH}_{2} \mathrm{Cl}_{2}(1 \mathrm{ml})$ at $0^{\circ} \mathrm{C}$ was added formic acid $(1 \mathrm{ml})$. The solution was stirred at $\mathrm{rt}$. for $16 \mathrm{~h}$ and toluene $(2 \mathrm{ml})$ was added. The mixture was concentrated to give crude diacid and purification by flash chromatography with $5 \% \mathrm{MeCN} / \mathrm{CH}_{2} \mathrm{Cl}_{2}$ as eluent afforded the diacid ent-3 (13 mg, 92\%) as a white gum. $[\alpha]_{\mathrm{D}}=+10.5$ (c 1.1, MeOH); Lit. $[\alpha]_{\mathrm{D}}=+9.4(c 10.85, \mathrm{MeOH}){ }^{1} \mathrm{H} \mathrm{NMR}\left(600 \mathrm{MHz}, d_{6}\right.$-acetone) $\delta 0.88(\mathrm{t}, J=7.0 \mathrm{~Hz}, 3 \mathrm{H}), 1.29-1.35$ $(\mathrm{m}, 12 \mathrm{H}), 2.04-2.06(\mathrm{~m}, 2 \mathrm{H}), 2.08-2.16(\mathrm{~m}, 5 \mathrm{H}), 2.41(\mathrm{~m}, 1 \mathrm{H}), 2.61(\mathrm{~s}, 1 \mathrm{H}), 3.02(\mathrm{dd}, J=10.0,6.2$ $\mathrm{Hz}, 1 \mathrm{H}), 3.03(\mathrm{~d}, J=16.6 \mathrm{~Hz}, 2 \mathrm{H}), 3.53$ (d, $J=16.6 \mathrm{~Hz}, 2 \mathrm{H}), 4.99$ (q, $J=7.1 \mathrm{~Hz}, 1 \mathrm{H}), 5.35-5.42(\mathrm{~m}$, $2 \mathrm{H}), 5.58(\mathrm{dd}, J=15.5,7.3 \mathrm{~Hz}, 1 \mathrm{H}), 5.81(\mathrm{dt}, J=15.4,6.5 \mathrm{~Hz}, 1 \mathrm{H}) .{ }^{13} \mathrm{C} \mathrm{NMR}\left(151 \mathrm{MHz}, \mathrm{CD}_{3} \mathrm{CN}\right) \delta$ 14.4, 23.4, 27.2, 27.9, 30.0, 30.03, 30.2, 30.4, 31.1, 32.6, 32.8, 40.8, 46.8, 76.0, 80.3, 129.3, 129.5, 131.5, 135.6, 172.3, 174.4, 176.1. ${ }^{13} \mathrm{C}$ NMR (151 MHz, $d_{6}$-acetone) $\delta 14.4,23.3,27.3,27.8,30.0$, 30.04, 30.4, 31.5, 32.6, 32.9, 41.4, 46.8, 75.9, 80.0, 129.4, 130.0, 131.2, 134.8, 172.3, 175.1, 175.7. IR (thin film): 968, 1187, 1729, 2854, 2924, 2956, $3256 \mathrm{~cm}^{-1}$. HRMS (ESI) calc. for $\mathrm{C}_{22} \mathrm{H}_{35} \mathrm{O}_{7}$ : $(\mathrm{M}+$ $\mathrm{H})^{+}, 411.2383$, found: $(\mathrm{M}+\mathrm{H})^{+}, 411.2380$.

\section{Citrafungin A tetra-t-butyl-ester (19)}

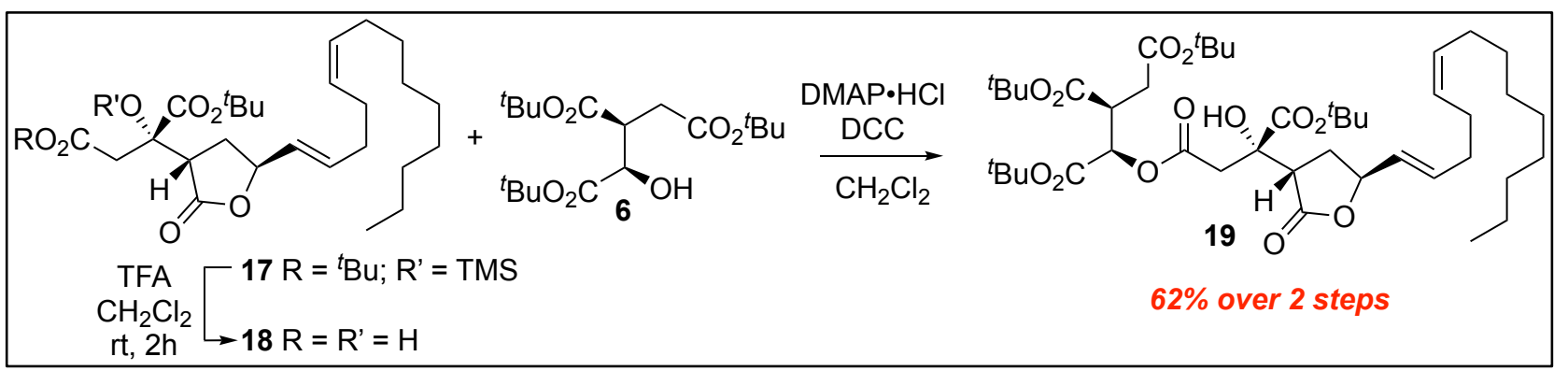

To a solution of the lactone $17(48 \mathrm{mg}, 0.08 \mathrm{mmol})$ in $\mathrm{CH}_{2} \mathrm{Cl}_{2}(4 \mathrm{~mL})$ at $0^{\circ} \mathrm{C}$ was added TFA $(300 \mu \mathrm{L})$. The solution was stirred at $0^{\circ} \mathrm{C}$ for $1 \mathrm{~h}$ then at $\mathrm{rt}$ for $2 \mathrm{~h}$ before toluene $(2 \mathrm{~mL})$ was added. The mixture was concentrated to give the crude mono acid 18 which was used directly to next reaction without further purification. To a solution of crude acid 18 and alcohol 6 (57 mg, $0.16 \mathrm{mmol}$ ) in $\mathrm{CH}_{2} \mathrm{Cl}_{2}$ $(500 \mu \mathrm{L})$ was added DMAP $\cdot \mathrm{HCl}(12 \mathrm{mg}, 0.08 \mathrm{mmol})$ at $0^{\circ} \mathrm{C}$. The reaction mixture was stirred for 10 min before DCC (33 mg, $0.16 \mathrm{mmol}$ ) was added, and the resulting solution was stirred at $\mathrm{rt}$ for $18 \mathrm{~h}$. The reaction mixture was concentrated and purification of the residue by flash chromatography using $10 \% \mathrm{EtOAc} /$ petrol as the eluent to afforded citrafungin A tetra-t-butyl ester (19) (40 $\mathrm{mg}, 62 \%)$ as a yellow oil. $[\alpha]_{\mathrm{D}}=+20.5\left(c 0.46, \mathrm{CH}_{2} \mathrm{Cl}_{2}\right) .{ }^{1} \mathrm{H} \mathrm{NMR}\left(500 \mathrm{MHz}, \mathrm{CDCl}_{3}\right) \delta 0.87(\mathrm{t}, J=6.9 \mathrm{~Hz}, 3 \mathrm{H})$, $1.26(\mathrm{~m}, 12 \mathrm{H}), 1.44(\mathrm{~s}, 18 \mathrm{H}), 1.46(\mathrm{~s}, 9 \mathrm{H}), 1.48(\mathrm{~s}, 9 \mathrm{H}), 1.96-2.02(\mathrm{~m}, 2 \mathrm{H}), 2.14-2.05(\mathrm{~m}, 4 \mathrm{H})$, $2.37-2.30(\mathrm{~m}, 1 \mathrm{H}), 2.38(\mathrm{dd}, J=16.9,5.0 \mathrm{~Hz}, 1 \mathrm{H}), 2.64(\mathrm{dd}, J=16.8,9.6 \mathrm{~Hz}, 1 \mathrm{H}), 2.88(\mathrm{~m}, 1 \mathrm{H})$, $2.91(\mathrm{~d}, J=15.4 \mathrm{~Hz}, 2 \mathrm{H}), 3.51(\mathrm{ddd}, J=9.2,5.1,3.8 \mathrm{~Hz}, 1 \mathrm{H}), 3.62(\mathrm{~d}, J=15.4 \mathrm{~Hz}, 2 \mathrm{H}), 4.40(\mathrm{~d}, J=$ $1.1 \mathrm{~Hz}, 1 \mathrm{H}), 5.01(\mathrm{q}, J=7.3 \mathrm{~Hz}, 1 \mathrm{H}), 5.21(\mathrm{~d}, J=3.2 \mathrm{~Hz}, 1 \mathrm{H}), 5.30-5.39(\mathrm{~m}, 2 \mathrm{H}), 5.43(\mathrm{dd}, J=$ 15.4, 7.5 Hz, 1H), $5.78(\mathrm{~m}, 1 \mathrm{H}) .{ }^{13} \mathrm{C} \mathrm{NMR}\left(151 \mathrm{MHz}, \mathrm{CDCl}_{3}\right) \delta 14.3,22.8,26.6,27.4,27.9,28.10$, 28.13, 28.2, 29.4, 29.5, 29.6, 29.8, 31.2, 32.0, 32.3, 33.5, 41.0, 43.7, 46.7, 72.5, 76.5, 80.2, 81.1, 82.3, 
83.6, 83.7, 128.2, 128.4, 131.0, 135.3, 166.9, 169.0, 169.9, 170.6, 171.5, 175.3. IR (thin film): 844, 1148, 1252, 1369, 1733, 2856, 2928, 2979, $3455 \mathrm{~cm}^{-1}$. HRMS (ESI) calc. for $\mathrm{C}_{44} \mathrm{H}_{72} \mathrm{O}_{13} \mathrm{Na}:(\mathrm{M}+$ $\mathrm{Na})^{+}, 831.4871$, found: $(\mathrm{M}+\mathrm{Na})^{+}, 831.4862$.

\section{Citrafungin A (1a)}

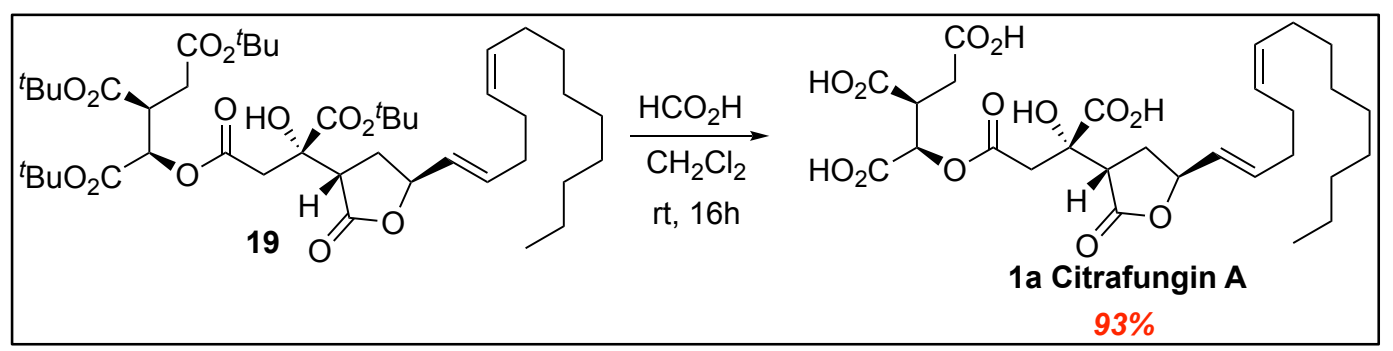

To a solution of the tetra-t-butyl ester $19(30 \mathrm{mg}, 0.037 \mathrm{mmol})$ in $\mathrm{CH}_{2} \mathrm{Cl}_{2}(0.5 \mathrm{ml})$ at $0^{\circ} \mathrm{C}$ was added formic acid $(0.5 \mathrm{ml})$. The solution was stirred at $\mathrm{rt}$ for $16 \mathrm{~h}$ and toluene $(2 \mathrm{ml})$ was then added. The mixture was concentrated to give citrafungin A $1 \mathbf{a}(20 \mathrm{mg}, 93 \%) .[\alpha]_{\mathrm{D}}=+24.3(c 0.75, \mathrm{MeOH}) .{ }^{1} \mathrm{H}$ NMR (500 MHz, $d_{6}$-acetone) $\delta 0.88(\mathrm{t}, J=7.0 \mathrm{~Hz}, 3 \mathrm{H}), 1.29-1.35(\mathrm{~m}, 12 \mathrm{H}), 2.05(\mathrm{q}, 6.5,1 \mathrm{H}), 2.11$ (m, 2H), $2.13(\mathrm{~m}, 2 \mathrm{H}), 2.40(\mathrm{ddd}, J=13.2,7.5,5.8 \mathrm{~Hz}, 1 \mathrm{H}), 2.62(\mathrm{dd}, J=17.3,5.0 \mathrm{~Hz}, 1 \mathrm{H}), 2.82(\mathrm{dd}$, $J=17.3,9.3 \mathrm{~Hz}, 1 \mathrm{H}), 3.08(\mathrm{dd}, J=9.7,6.0 \mathrm{~Hz}, 1 \mathrm{H}), 3.15(\mathrm{~d}, J=16.4 \mathrm{~Hz}, 1 \mathrm{H}), 3.58$ (ddd, $J=9.0,5.0$, $3.6 \mathrm{~Hz}, 1 \mathrm{H}), 3.69$ (d, $J=16.4 \mathrm{~Hz}, 1 \mathrm{H}), 5.00$ (q, $J=7.0 \mathrm{~Hz}, 1 \mathrm{H}), 5.34(\mathrm{~m}, 1 \mathrm{H}), 5.37$ (m, 1H), 5.49 (d, $J=3.6 \mathrm{~Hz}, 1 \mathrm{H}), 5.59(\mathrm{dd}, J=15.4,7.4 \mathrm{~Hz}, 1 \mathrm{H}), 5.82(\mathrm{~m}, 1 \mathrm{H}) .{ }^{13} \mathrm{C}$ NMR $\left(151 \mathrm{MHz}, d_{6}\right.$-acetone, highest peak set to $175.0 \mathrm{ppm}) \delta 13.8,22.8,26.7,27.3,29.4,29.5,29.7,29.9,30.9,31.7,32.1,32.3$, 40.9, 42.7, 46.4, 72.0, 75.6, 79.5, 128.8, 129.3, 130.7, 134.4, 168.7, 169.5, 170.9, 172.2, 173.6, 175.0 . ${ }^{3} \mathrm{C}$ NMR (151 MHz, $d_{6}$-acetone set to $\left.29.8 \mathrm{ppm}\right) 14.4,23.3,27.3,27.8,29.98,30.02,30.2,30.4,31.5$, $32.3,32.6,32.9,41.4,43.3,46.9,72.5,76.2,80.1,129.4,129.8,131.2,135.0,169.2,170.1,171.5$, 172.8, 174.2, 175.5. IR (thin film): 966, 1179, 1721, 2854, 2924, $3451 \mathrm{~cm}^{-1}$. HRMS (ESI) calc. for $\mathrm{C}_{28} \mathrm{H}_{41} \mathrm{O}_{13}:(\mathrm{M}+\mathrm{H})^{+}, 585.2548$, found: $(\mathrm{M}+\mathrm{H})^{+}, 585.2545$. 


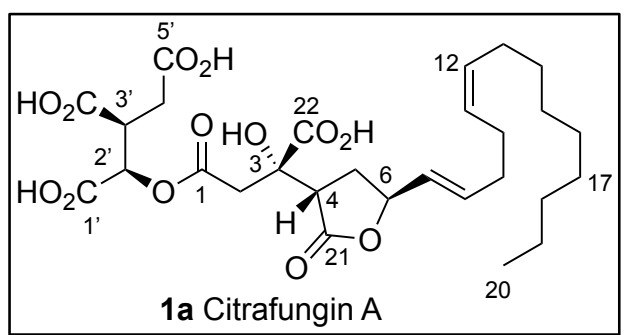

\begin{tabular}{|c|c|c|c|}
\hline H atom & Nat. $^{4} \delta, m, J$ Hz & Syn. 1a $\delta, \mathbf{m}, J$ Hz & Syn. $1^{1} \delta, m, J$ Hz \\
\hline $\mathbf{H} \mathbf{2}_{\mathbf{a}}$ & $3.66, d, 16$ & $3.68, \mathrm{~d}, 16.4$ & $3.70, \mathrm{~d}, 16.3$ \\
\hline $\mathbf{H} \mathbf{2}_{\mathrm{b}}$ & $3.13, \mathrm{~d}, 16.5$ & $3.15 . \mathrm{d}, 16.4$ & $3.16, \mathrm{~d}, 16.3$ \\
\hline H4 & $3.07, \mathrm{dd}, 10,6$ & $3.08, \mathrm{dd}, 9.7,6.0$ & $3.05, \mathrm{dd}, 9.7,5.8$ \\
\hline H5a & 2.39, ddd, $13,7.5,6.0$ & $2.40, \mathrm{ddd}, 13.4,7.6,6.0$ & 2.39 , ddd, $13.2,7.4,6.0$ \\
\hline H5 & $2.11, \mathrm{~m}$ & $2.13, \mathrm{~m}$ & $2.16-2.03, \mathrm{~m}$ \\
\hline H6 & $4.99, \mathrm{q}, 7$ & $5.00, \mathrm{q}, 7.0$ & $4.99, \mathrm{q}, 7.1$ \\
\hline H7 & $5.57, \mathrm{dd}, 15,7.5$ & $5.59, \mathrm{dd}, 15.4,7.4$ & $5.58, \mathrm{dd}, 15.4,7.4$ \\
\hline H8 & $5.81, \mathrm{dt}, 15.5,7$ & $5.82, \mathrm{dt}, 15.3,6.2$ & $5.82, \mathrm{dt}, 14.9,6.3$ \\
\hline $\mathbf{H} 9_{\mathbf{a b}}$ & $2.11, \mathrm{~m}$ & $2.11, \mathrm{~m}$ & $2.16-2.03$ \\
\hline H10 ab & $2.13, \mathrm{~m}$ & $2.13, \mathrm{~m}$ & $2.16-2.03$ \\
\hline H11 & $5.34, \mathrm{~m}$ & $5.34, \mathrm{~m}$ & $5.41-5.33, \mathrm{~m}$ \\
\hline H12 & $5.37, \mathrm{~m}$ & $5.37, \mathrm{~m}$ & $5.41-5.33, \mathrm{~m}$ \\
\hline $\mathbf{H 1 3}_{\mathbf{a b}}$ & $2.04, \mathrm{q}, 6.5$ & $2.05, \mathrm{q}, 6.5$ & $2.16-2.03, \mathrm{~m}$ \\
\hline H14-19 & $1.28, \mathrm{~m}$ & $1.29-1.35, \mathrm{~m}$ & $1.28, \mathrm{~m}$ \\
\hline H20 & $0.86, \mathrm{t}, 7$ & $0.88, \mathrm{t}, 7.0$ & $0.87, \mathrm{t}, 6.9$ \\
\hline H2' & $5.47, \mathrm{~d}, 3.5$ & $5.49, \mathrm{~d}, 3.6 \mathrm{~Hz}$ & $5.49, \mathrm{~d}, 3.4$ \\
\hline H3' & 3.56 , ddd, $8.5,5.0,3.5$ & $3.58, \mathrm{ddd}, 9.0,5.0,3.6$ & 3.56, ddd, $8.8,4.8,5.7$ \\
\hline H4' & $2.80, \mathrm{dd}, 17.5,9.5$ & $2.82, \mathrm{dd}, 17.3,9.3$ & $2.81, \mathrm{dd}, 17.2,9.4$ \\
\hline H4' ${ }_{b}$ & $2.60, \mathrm{dd}, 17,4.5$ & $2.62, \mathrm{dd}, 17.3,5.0$ & $2.61, \mathrm{dd}, 17.2,4.9$ \\
\hline
\end{tabular}

Table 1: Comparison of ${ }^{1} \mathrm{H}$ spectral data for natural ${ }^{4}\left(600 \mathrm{MHz}, d_{6}\right.$-acetone) and synthetic citrafungin A (1a) $\left(500 \mathrm{MHz}, d_{6}\right.$-acetone) and synthetic $1^{1}$ (600 MHz, $d_{6}$-acetone). 


\begin{tabular}{|c|c|c|c|c|c|}
\hline Carbon & Nat. $^{\mathbf{4}}$ & Syn. 1a & Syn. $\mathbf{1}^{\mathbf{1}}$ & Nat. - 1a & Nat. - 1 \\
\hline $\mathbf{1}$ & 169.4 & 169.5 & 169 & -0.1 & 0.4 \\
\hline $\mathbf{2}$ & 40.7 & 40.9 & 40.8 & -0.2 & -0.1 \\
\hline $\mathbf{3}$ & 75.5 & 75.6 & 75.6 & -0.1 & -0.1 \\
\hline $\mathbf{4}$ & 46.3 & 46.4 & 46.3 & -0.1 & 0 \\
\hline $\mathbf{5}$ & 30.8 & 30.9 & 30.9 & -0.1 & -0.1 \\
\hline $\mathbf{6}$ & 79.4 & 79.5 & 79.5 & -0.1 & -0.1 \\
\hline $\mathbf{7}$ & 129.1 & 129.3 & 129.2 & -0.2 & -0.1 \\
\hline $\mathbf{8}$ & 134.4 & 134.4 & 134.4 & 0 & 0 \\
\hline $\mathbf{9}$ & 32.2 & 32.3 & 32.3 & -0.1 & -0.1 \\
\hline $\mathbf{1 0}$ & 26.6 & 26.7 & 26.7 & -0.1 & -0.1 \\
\hline $\mathbf{1 1}$ & 128.7 & 128.8 & 128.8 & -0.1 & -0.1 \\
\hline $\mathbf{1 2}$ & 130.6 & 130.7 & 130.6 & -0.1 & 0 \\
\hline $\mathbf{1 3}$ & 27.2 & 27.3 & 27.2 & -0.1 & 0 \\
\hline $\mathbf{1 4}$ & 29.7 & 29.9 & 30.3 & -0.2 & -0.6 \\
\hline $\mathbf{1 5}$ & 29.6 & 29.7 & - & -0.1 & - \\
\hline $\mathbf{1 6}$ & 29.5 & 29.5 & - & 0 & - \\
\hline $\mathbf{1 7} *$ & $28.8 *$ & 29.4 & - & $-0.6 *$ & - \\
\hline $\mathbf{1 8}$ & 31.9 & 31.7 & 31.8 & 0.2 & 0.1 \\
\hline $\mathbf{1 9}$ & 22.7 & 22.8 & 22.7 & -0.1 & 0 \\
\hline $\mathbf{2 0}$ & 13.8 & 13.8 & 13.8 & 0 & 0 \\
\hline $\mathbf{2 1}$ & 175 & 175 & 175 & 0 & 0 \\
\hline $\mathbf{2 2}$ & 173.6 & 173.6 & 173.8 & 0 & -0.2 \\
\hline $\mathbf{1}$ & 168.7 & 168.7 & 169.2 & 0 & -0.5 \\
\hline $\mathbf{2}$ & 71.9 & 72 & 71.9 & -0.1 & 0 \\
\hline $\mathbf{3}$ & 42.6 & 42.7 & 42.7 & -0.1 & -0.1 \\
\hline $\mathbf{4}^{\prime}$ & 31.6 & 31.7 & 31.8 & -0.1 & -0.2 \\
\hline $\mathbf{5}$ & 172.3 & 172.2 & 172.3 & 0.1 & 0 \\
\hline $\mathbf{6}$ & 170.9 & 170.9 & 171.1 & 0 & -0.2 \\
\hline $\mathbf{1}$ & & & & & 0.1 \\
\hline
\end{tabular}

Table 2: ${ }^{13} \mathrm{C}$ NMR chemical shift data and shift differences for natural ${ }^{4}$ citrafungin $\mathrm{A}(150 \mathrm{MHz}$, $d_{6}$-acetone) and synthetic $\mathbf{1 a}\left(126 \mathrm{MHz}, d_{6}\right.$-acetone) and synthetic $\mathbf{1}^{1}$ (75 MHz, $d_{6}$-acetone).

*Misassigned in isolation paper ${ }^{4}$

\section{References}

(1) Calo, F.; Richardson, J.; Barrett, A. J. Org. Chem. 2008, 73, 9692-9697.

(2) Chen, Y. K.; Jeon, S.-J.; Walsh, P. J.; Nugent, W. A. Org. Synth. 2005, 82, 87-88.

(3) Tsegay, S.; Hugel, A. H.; Rizzacasa, M. A. Aust. J. Chem. 2009, 72, 676-682.

(4) Singh, S. B.; Zink, D. L.; Doss, G. A.; J. D. Polishook; Ruby, C.; Register, E.; Kelly, T. M.; C. Bonfiglio; Williamson, J. M.; Kelly, R. Org. Lett. 2004, 6, 337-340. 
${ }^{1} \mathrm{H}$ NMR spectrum (400 MHz, $\mathrm{CDCl}_{3}$ )
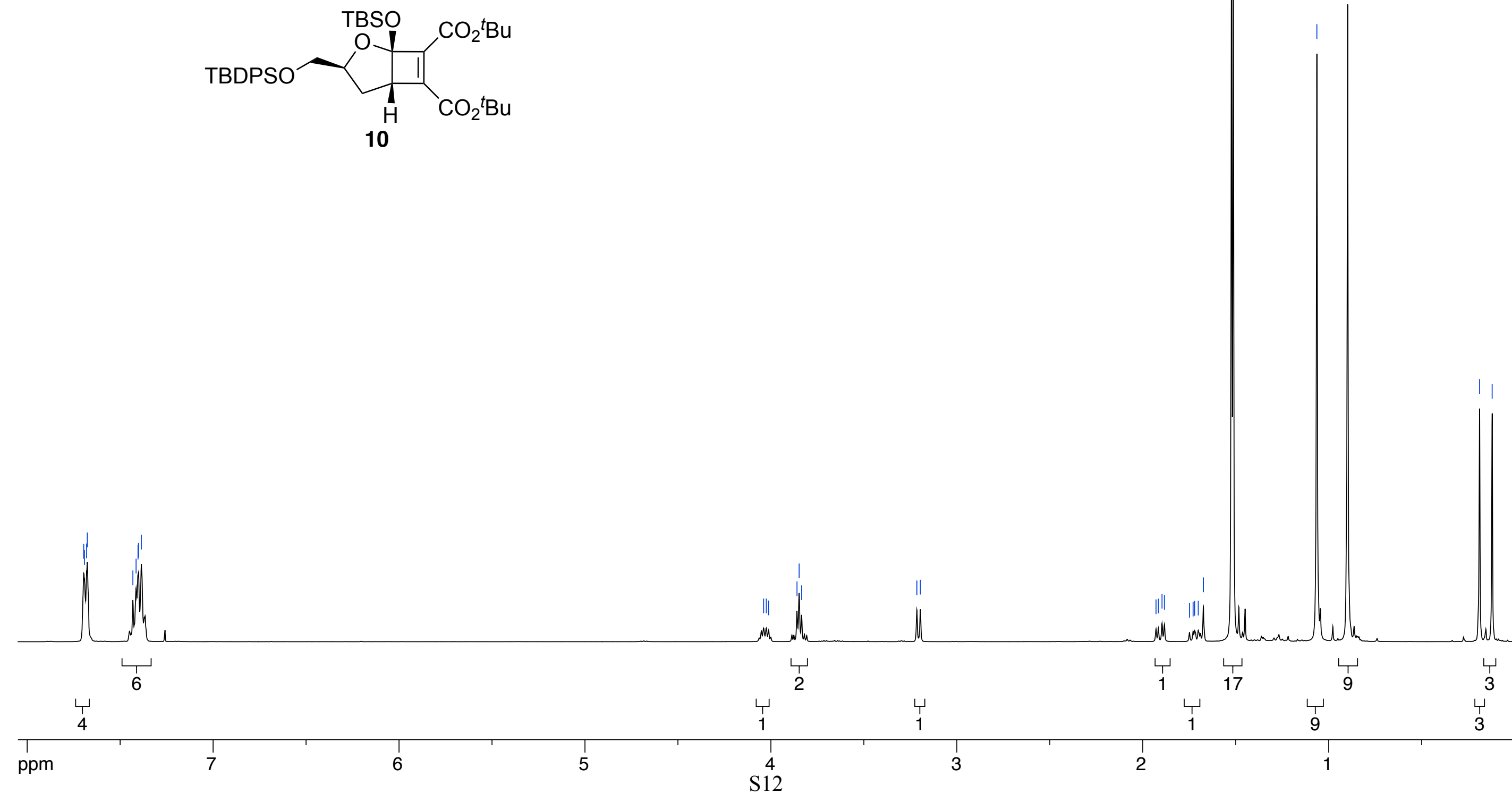
${ }^{13} \mathrm{C}$ NMR spectrum (100 MHz, $\mathrm{CDCl}_{3}$ )

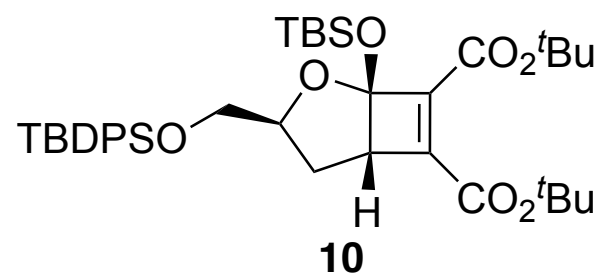

10

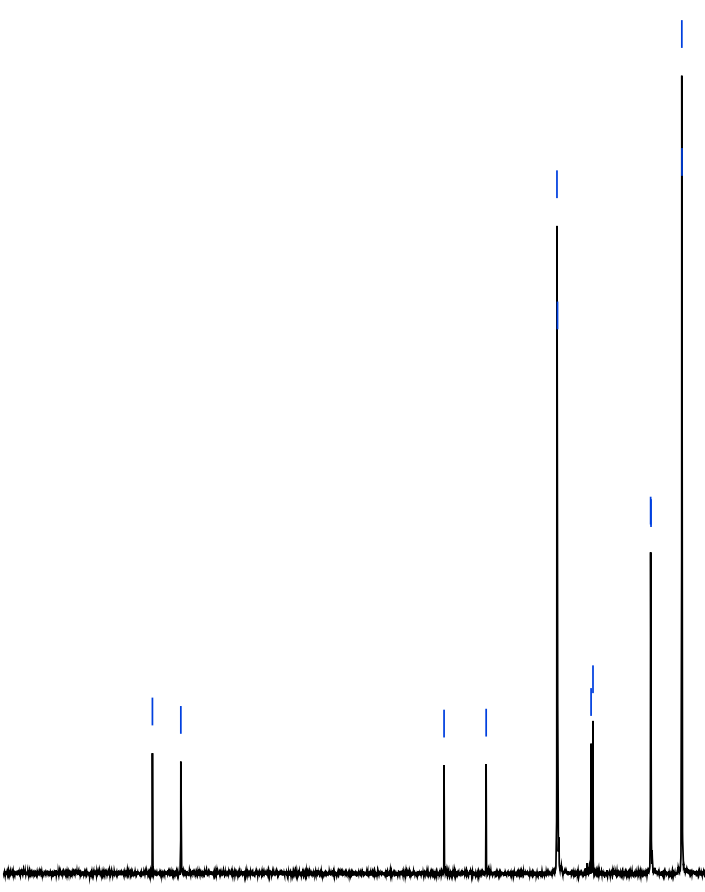



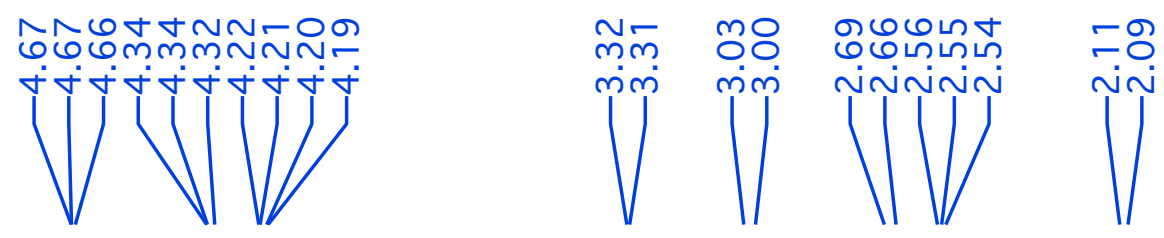

${ }^{1} \mathrm{H}$ NMR spectrum (400 MHz, $\mathrm{CDCl}_{3}$ )
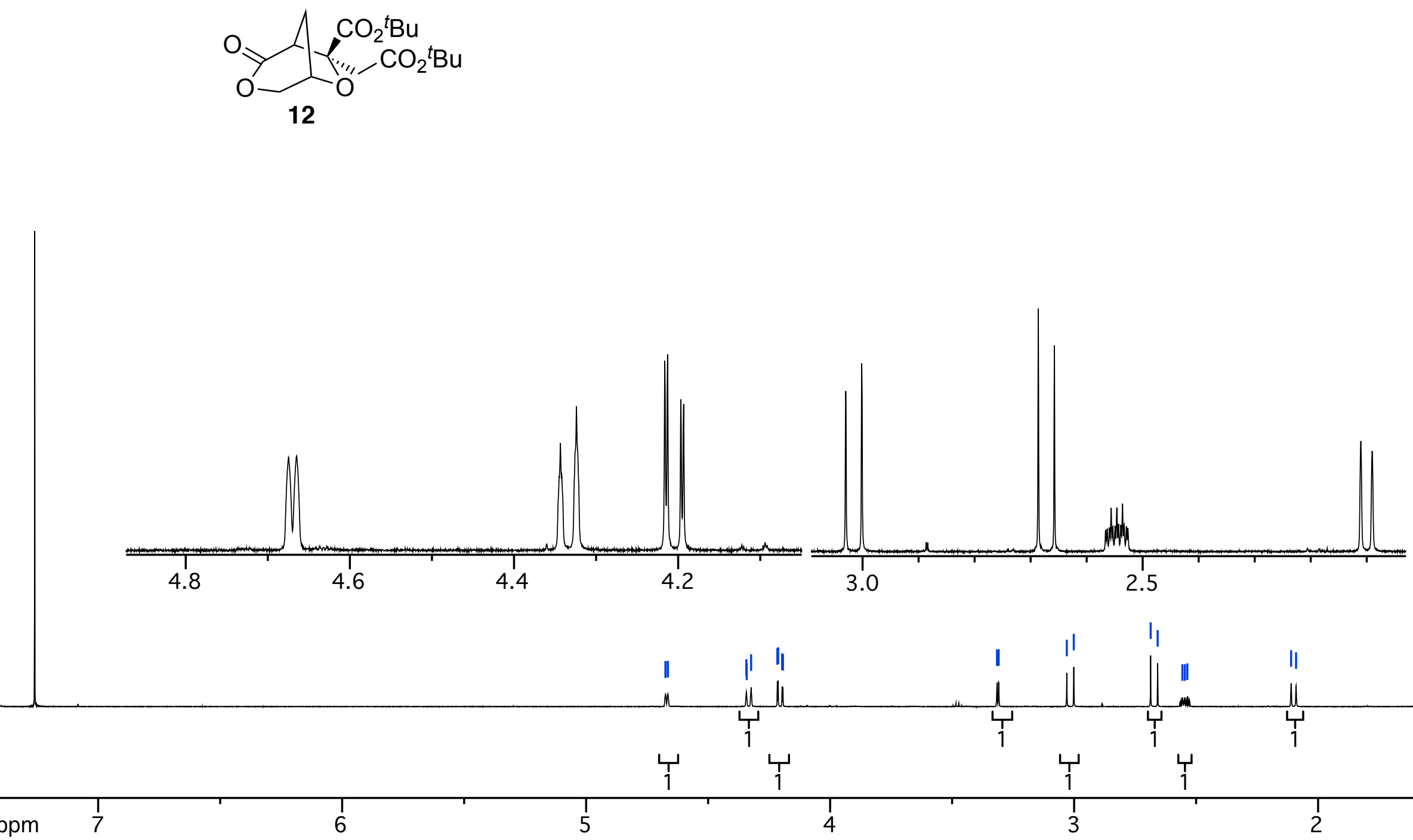

بิ 
${ }^{13} \mathrm{C}$ NMR spectrum (101 $\mathrm{MHz}, \mathrm{CDCl}_{3}$ )

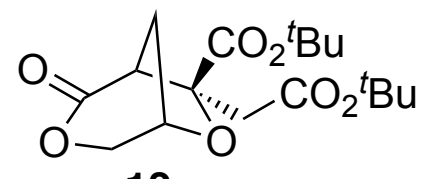

12

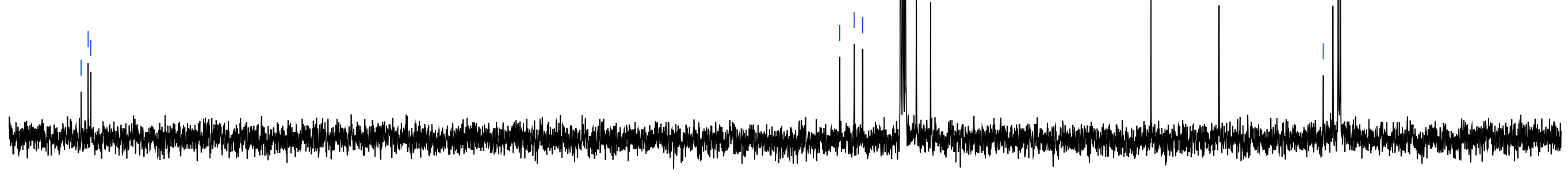




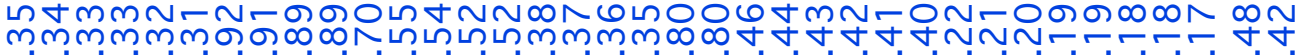

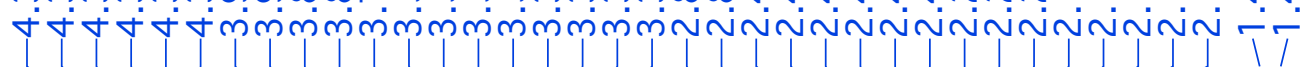

${ }^{1} \mathrm{H}$ NMR spectrum $\left(500 \mathrm{MHz}, \mathrm{CDCl}_{3}\right)$
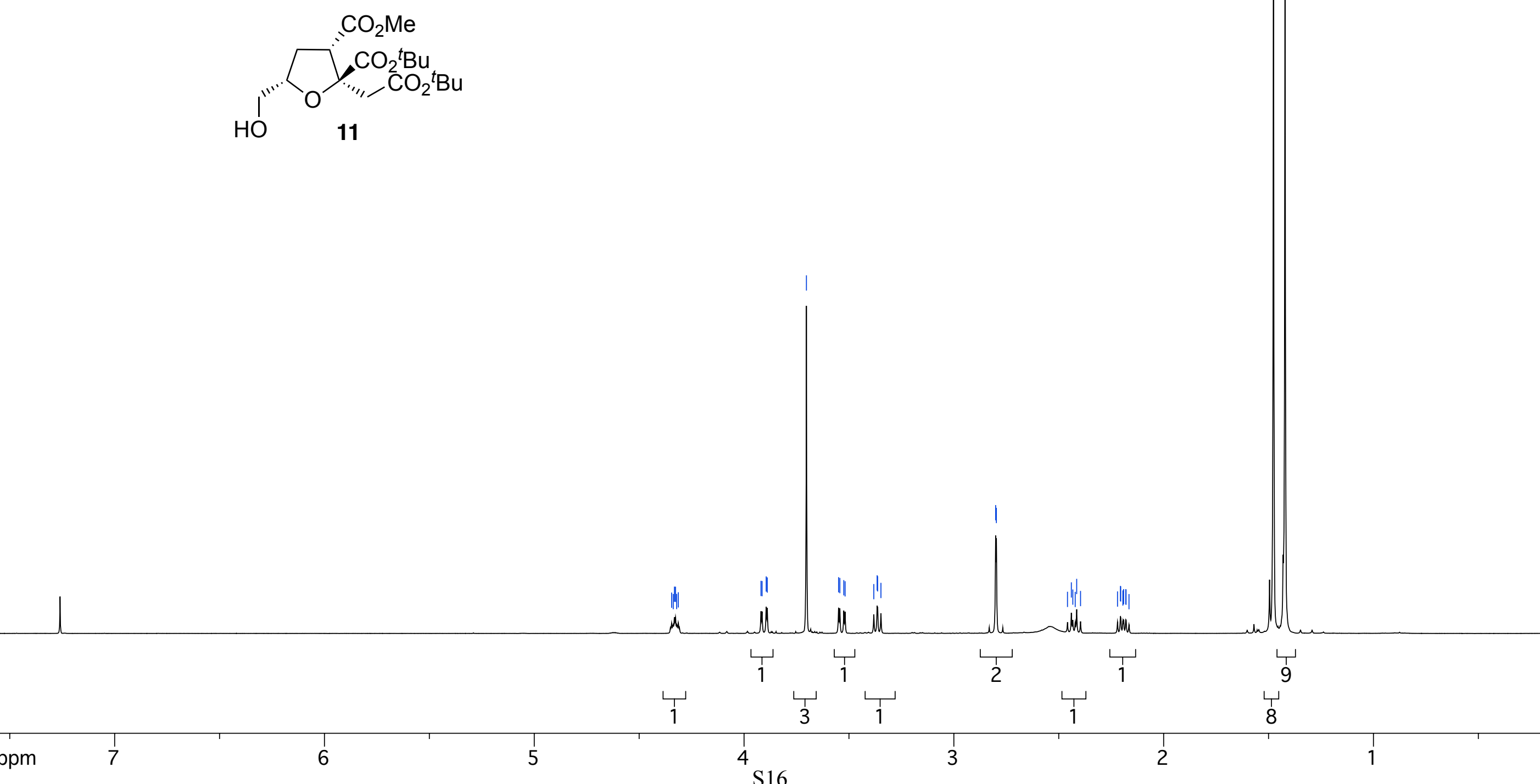
${ }^{13} \mathrm{C}$ NMR spectrum (125 $\mathrm{MHz}, \mathrm{CDCl}_{3}$ )
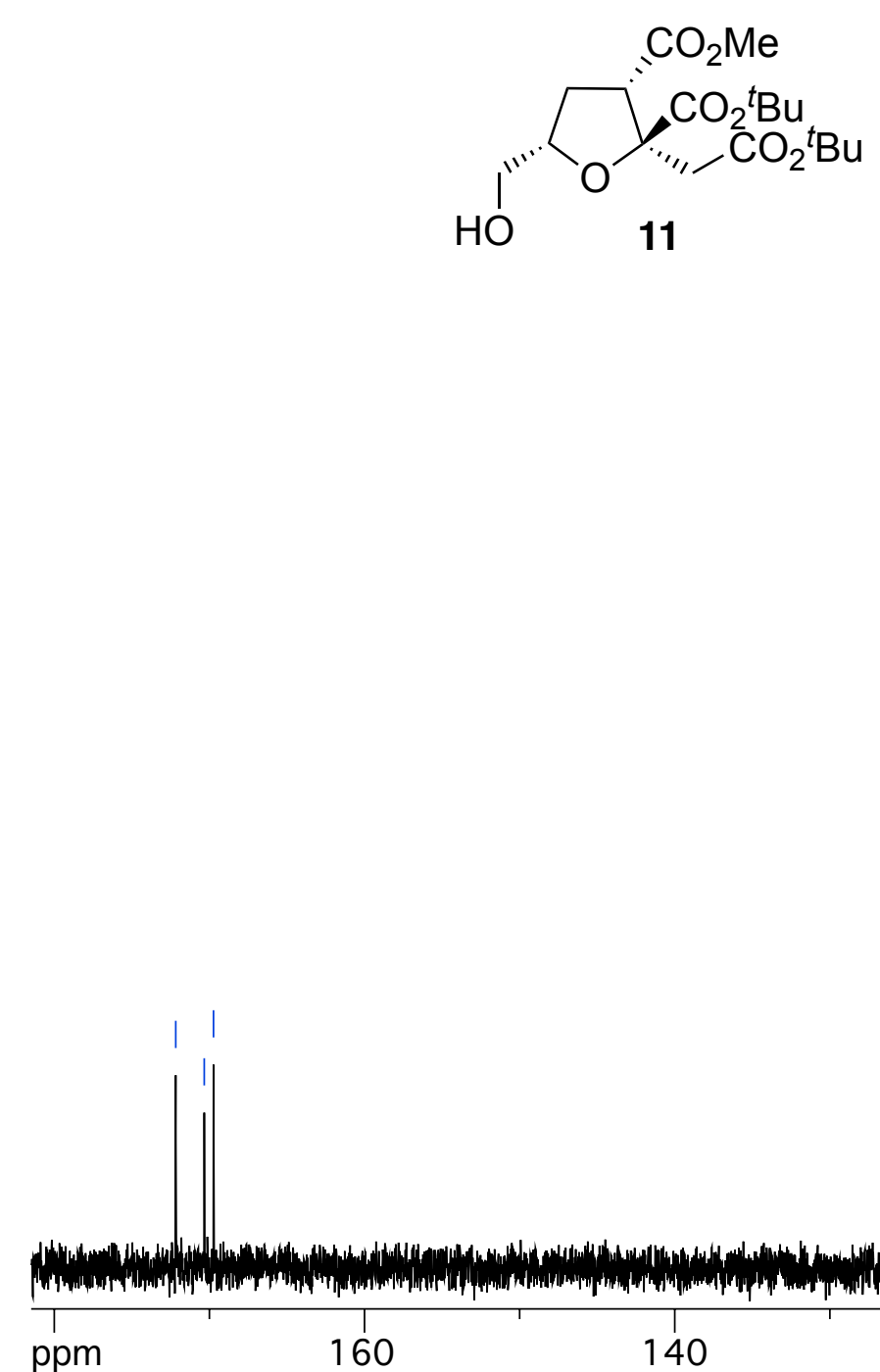

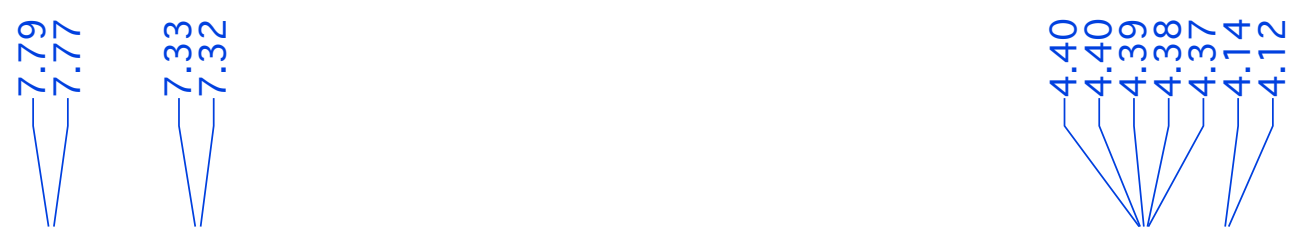

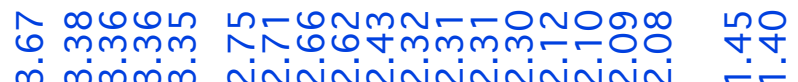

${ }^{1} \mathrm{H}$ NMR spectrum $\left(500 \mathrm{MHz}, \mathrm{CDCl}_{3}\right)$

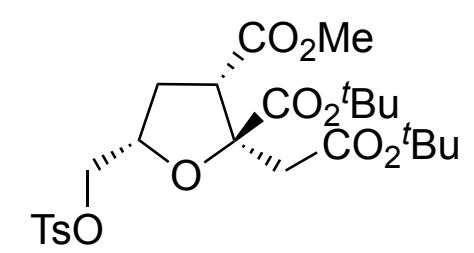

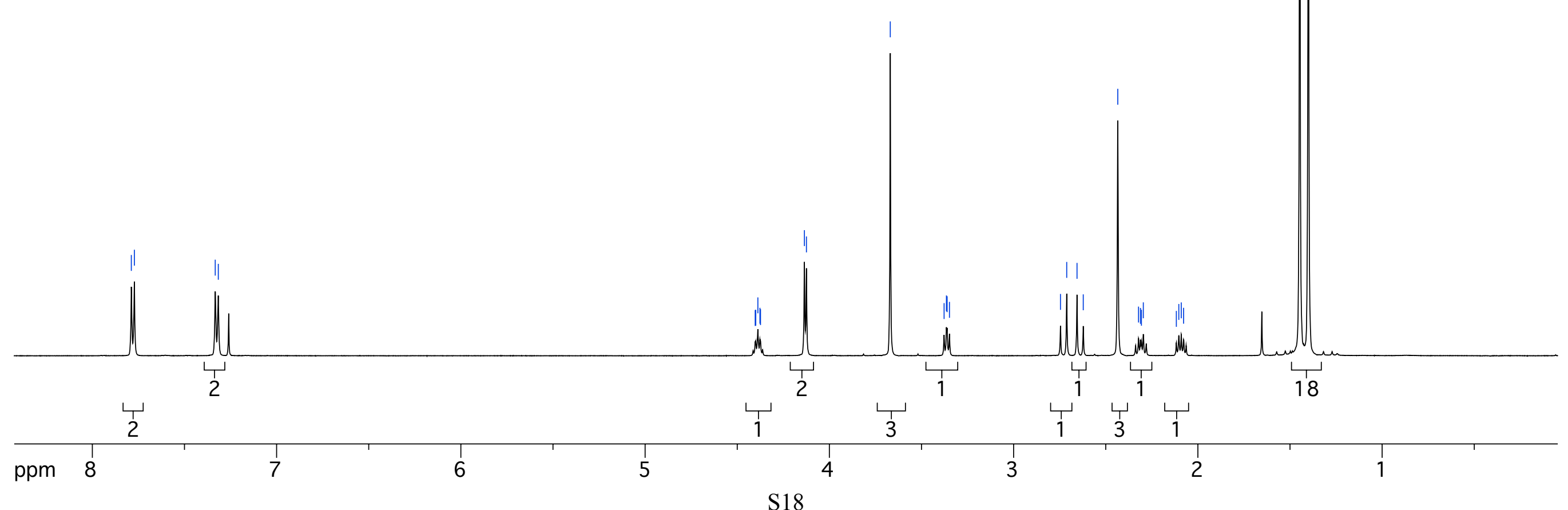




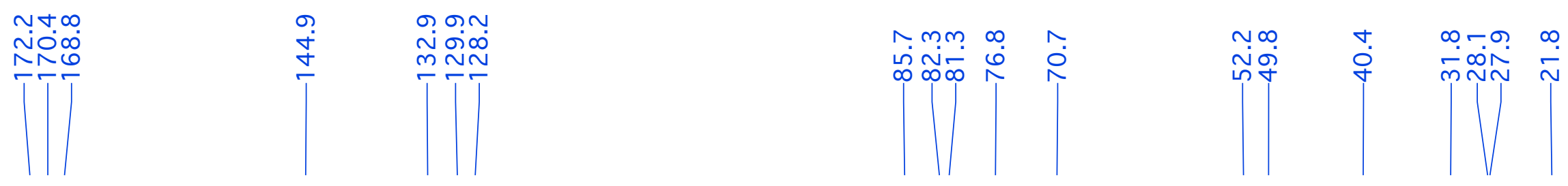

${ }^{13} \mathrm{C}$ NMR spectrum (125.5 MHz, $\mathrm{CDCl}_{3}$ )

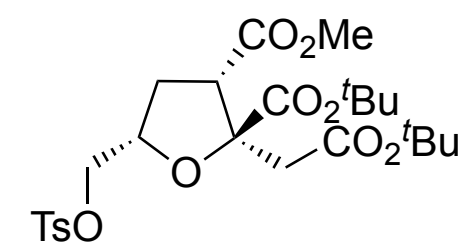




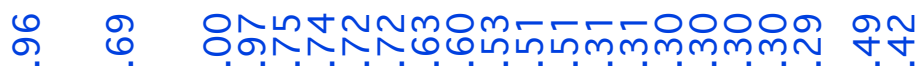

mNNNNNNNNNNNNNNNO

${ }^{1} \mathrm{H}$ NMR spectrum $\left(500 \mathrm{MHz}, \mathrm{CDCl}_{3}\right)$
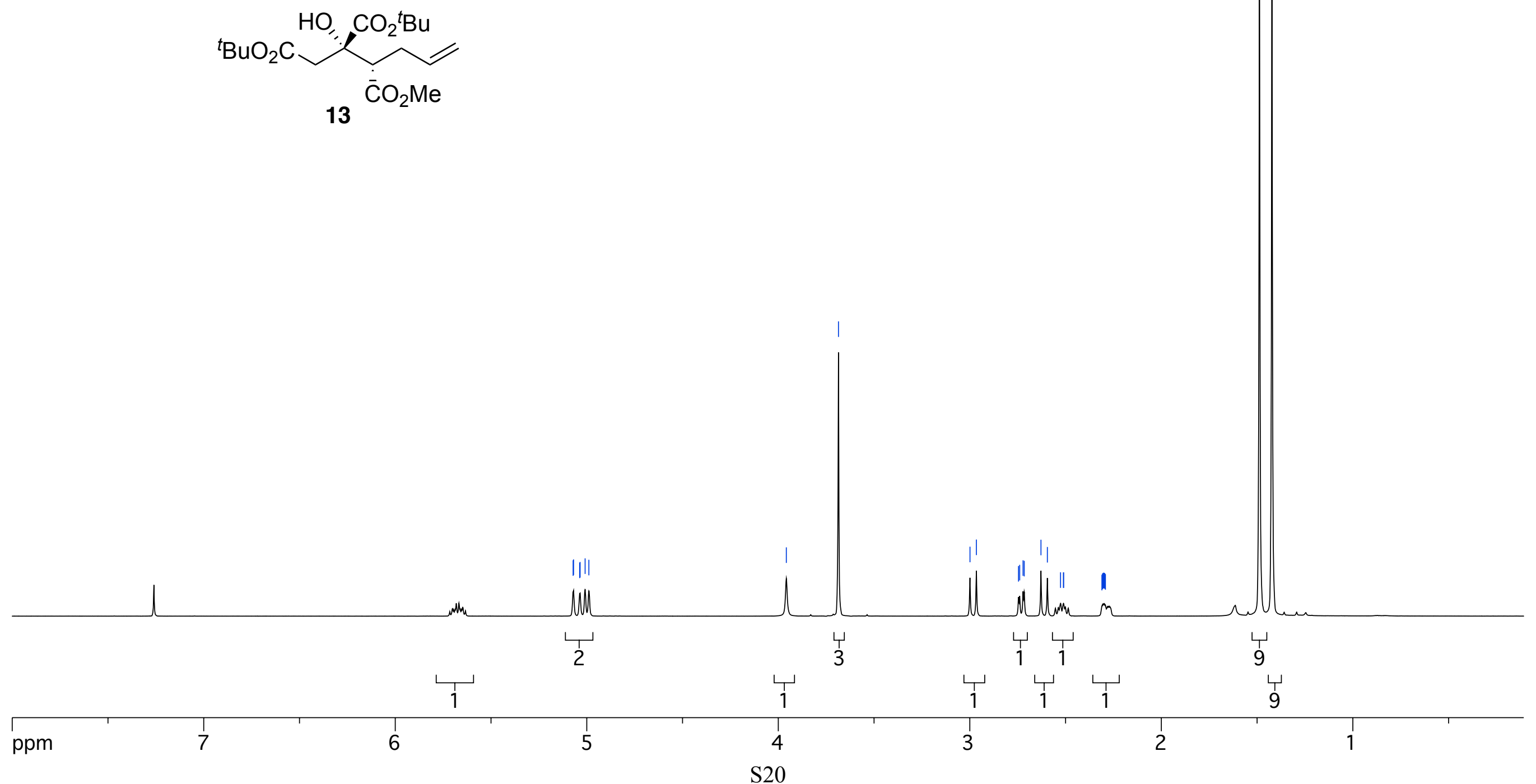


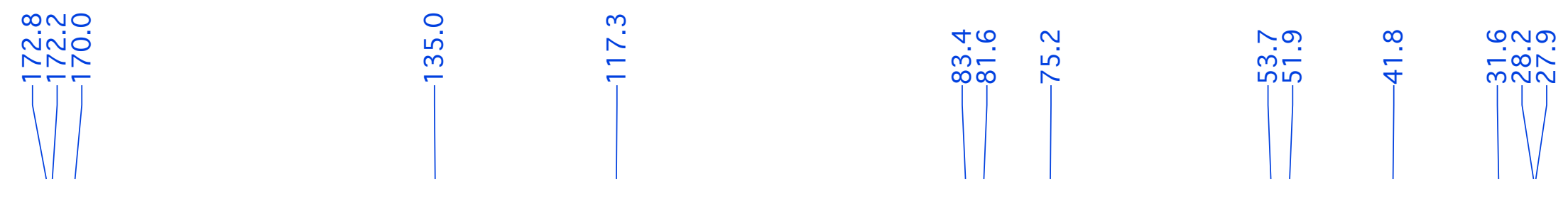

${ }^{13} \mathrm{C}$ NMR spectrum (125.5 MHz, $\mathrm{CDCl}_{3}$ )
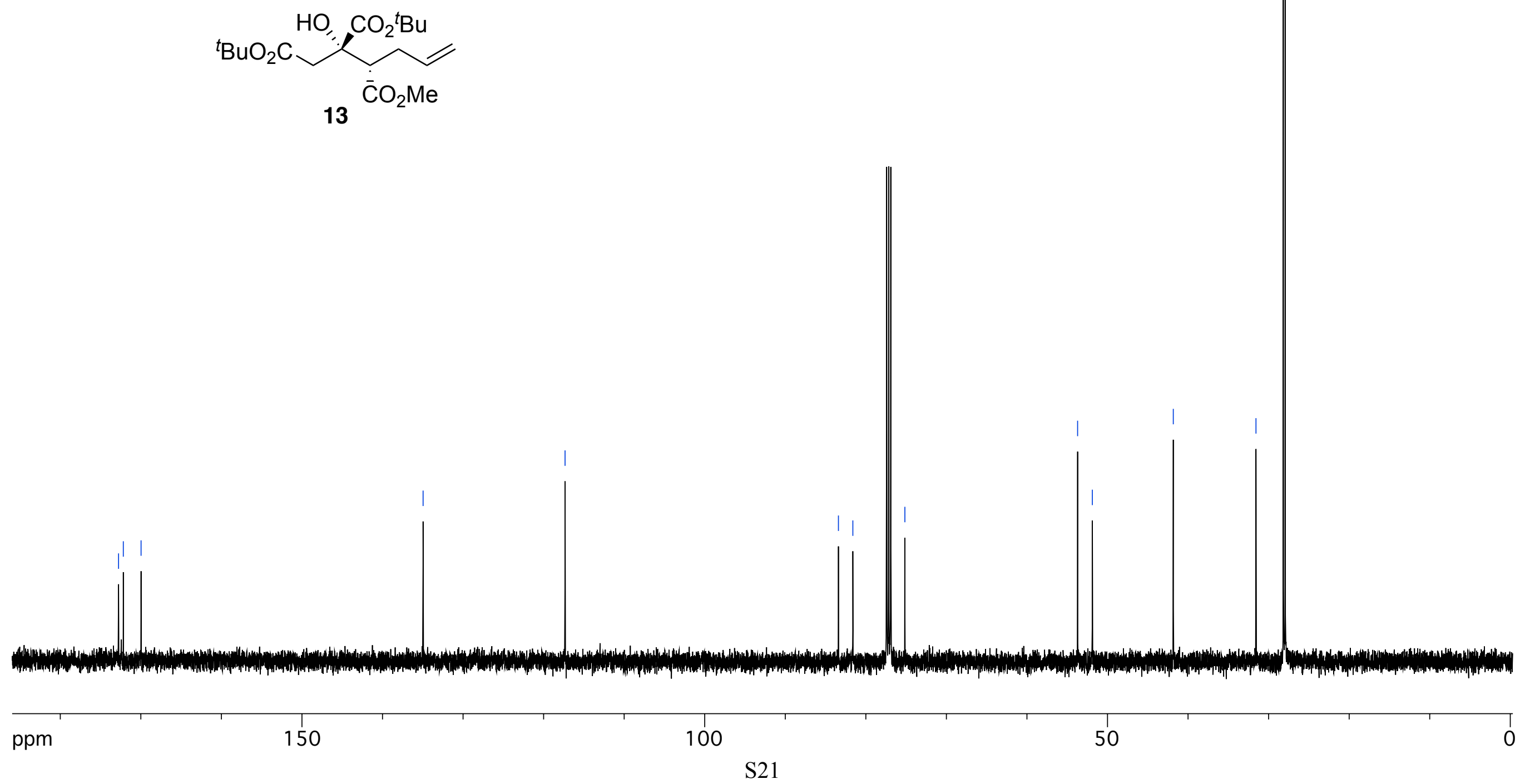
Г⿸丆⿰丨丶刀

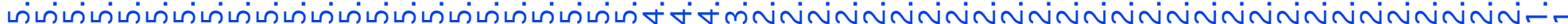
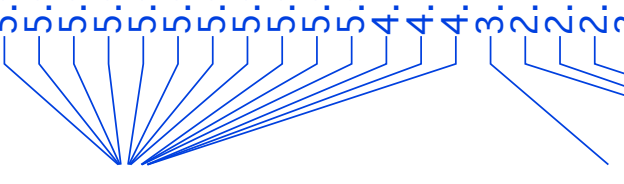

${ }^{1} \mathrm{H}$ NMR spectrum $\left(600 \mathrm{MHz}, \mathrm{CDCl}_{3}\right)$

$$
14 \underbrace{{ }^{t} \mathrm{BuO}_{2} \mathrm{C}}_{\overline{\mathrm{C}} \mathrm{O}_{2} \mathrm{Me}}
$$

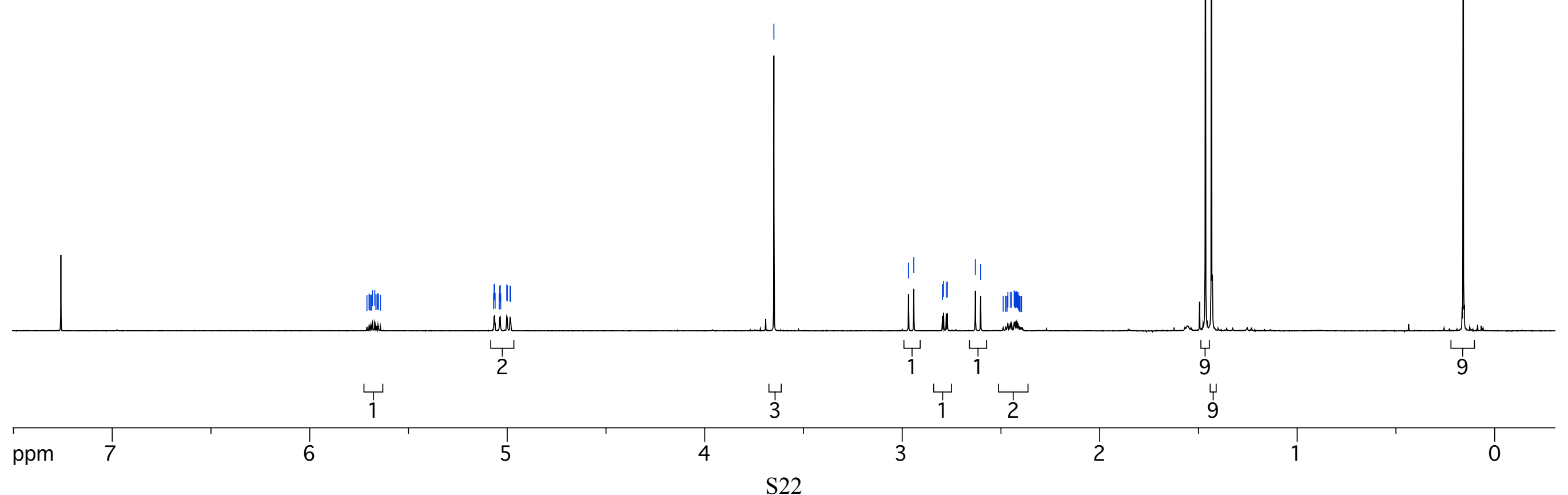


${ }^{13} \mathrm{C}$ NMR spectrum (151 $\mathrm{MHz}, \mathrm{CDCl}_{3}$ )

$$
{ }_{14}^{{ }_{\mathrm{BuO}} \mathrm{C}} \underbrace{\mathrm{TMSO}}_{\mathrm{\overline {C }}_{2} \mathrm{Me}} \underbrace{\mathrm{CO}_{2}{ }^{\mathrm{B}} \mathrm{Bu}}
$$




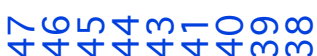

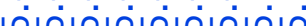

(L)

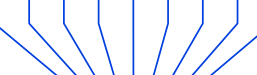

'H NMR spectrum $\left(600 \mathrm{MHz}, \mathrm{CDCl}_{3}\right)$

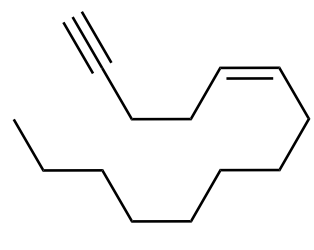

8

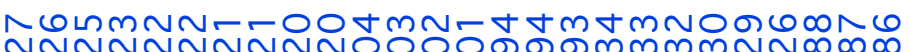
Ninininininininininits NNNNNNNNNNNNNN-T-T-T-000
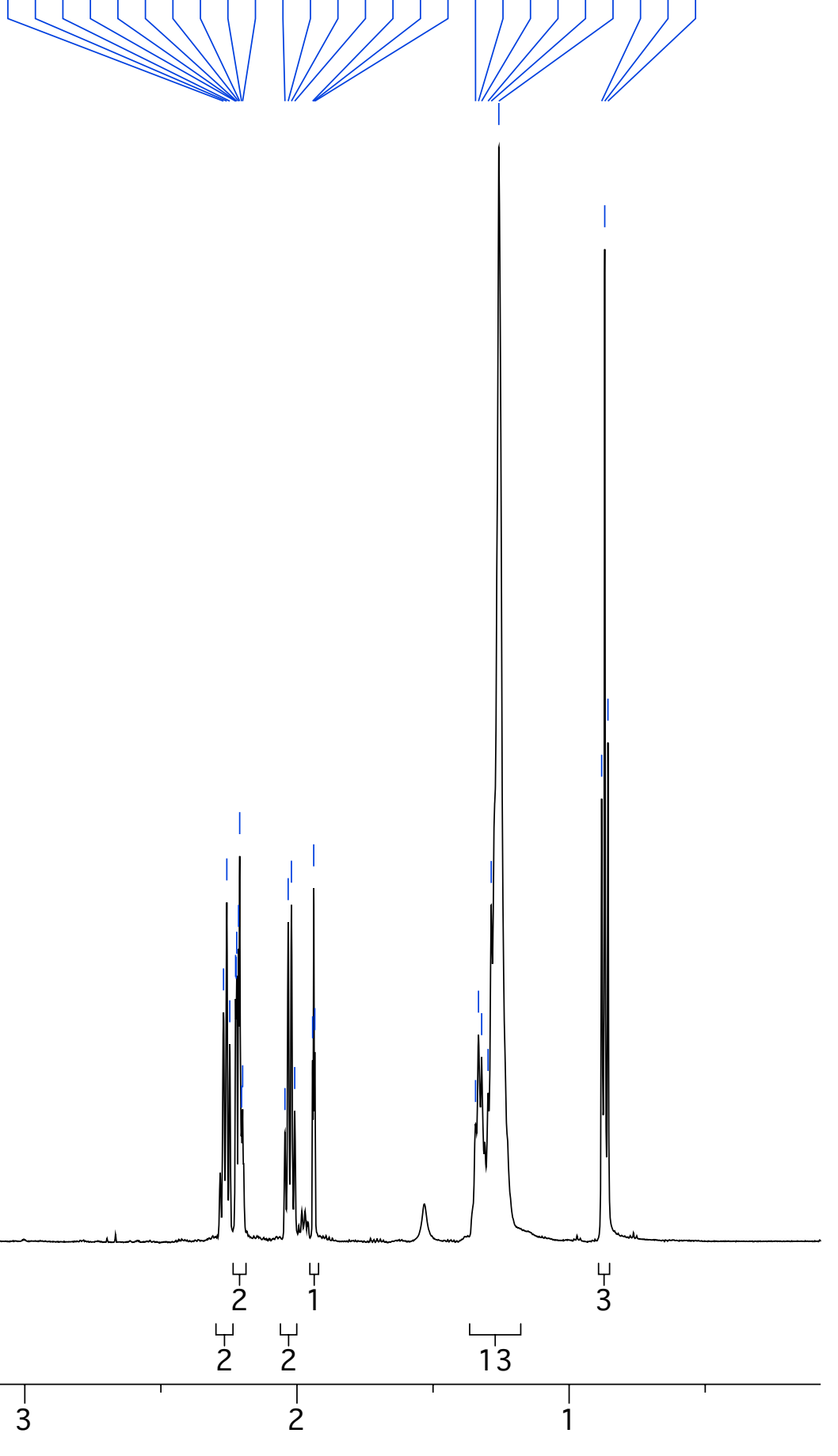

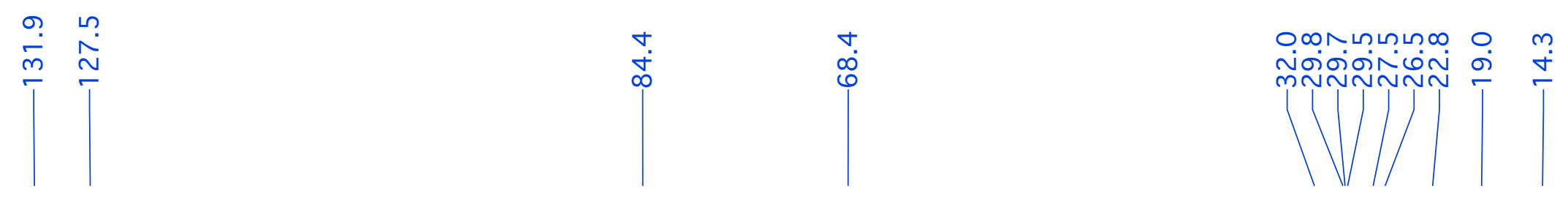

${ }^{13} \mathrm{C}$ NMR spectrum (151 MHz, $\mathrm{CDCl}_{3}$ )

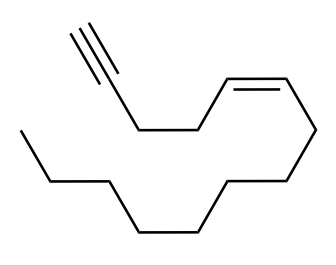

8

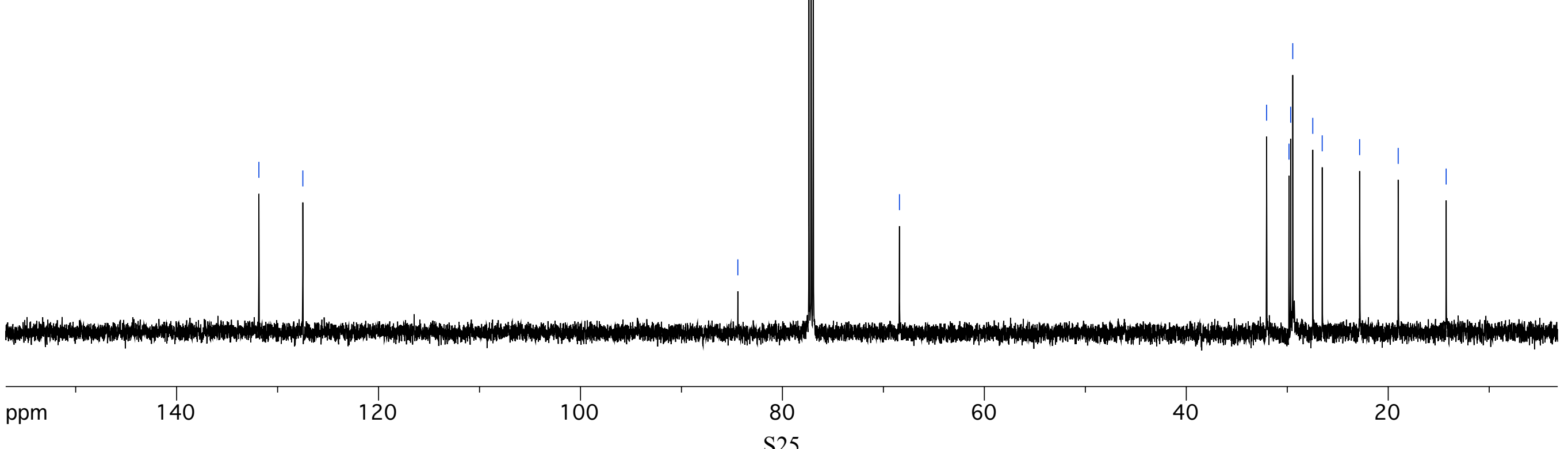


${ }^{1} \mathrm{H}$ NMR spectrum $\left(500 \mathrm{MHz}, \mathrm{CDCl}_{3}\right)$

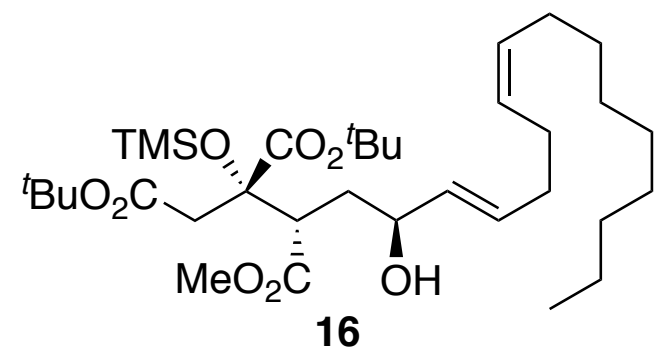

16 


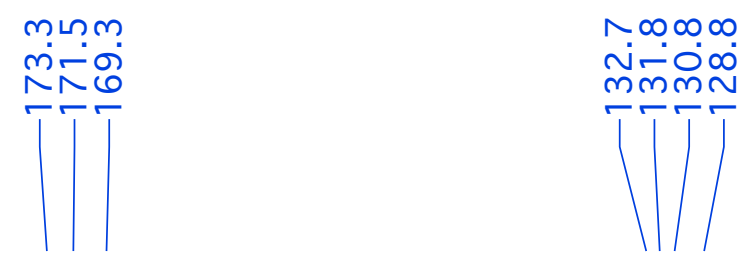

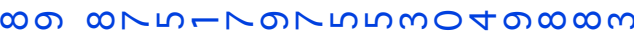

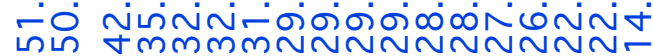

${ }^{13} \mathrm{C}$ NMR spectrum (125 $\mathrm{MHz}, \mathrm{CDCl}_{3}$ )

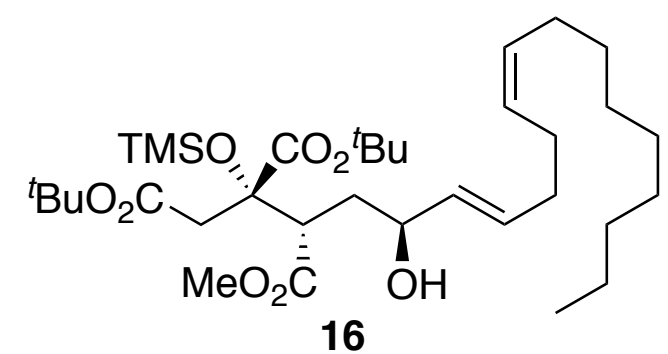

16

II 
${ }^{1} \mathrm{H}$ NMR spectrum $\left(500 \mathrm{MHz}, \mathrm{CDCl}_{3}\right)$
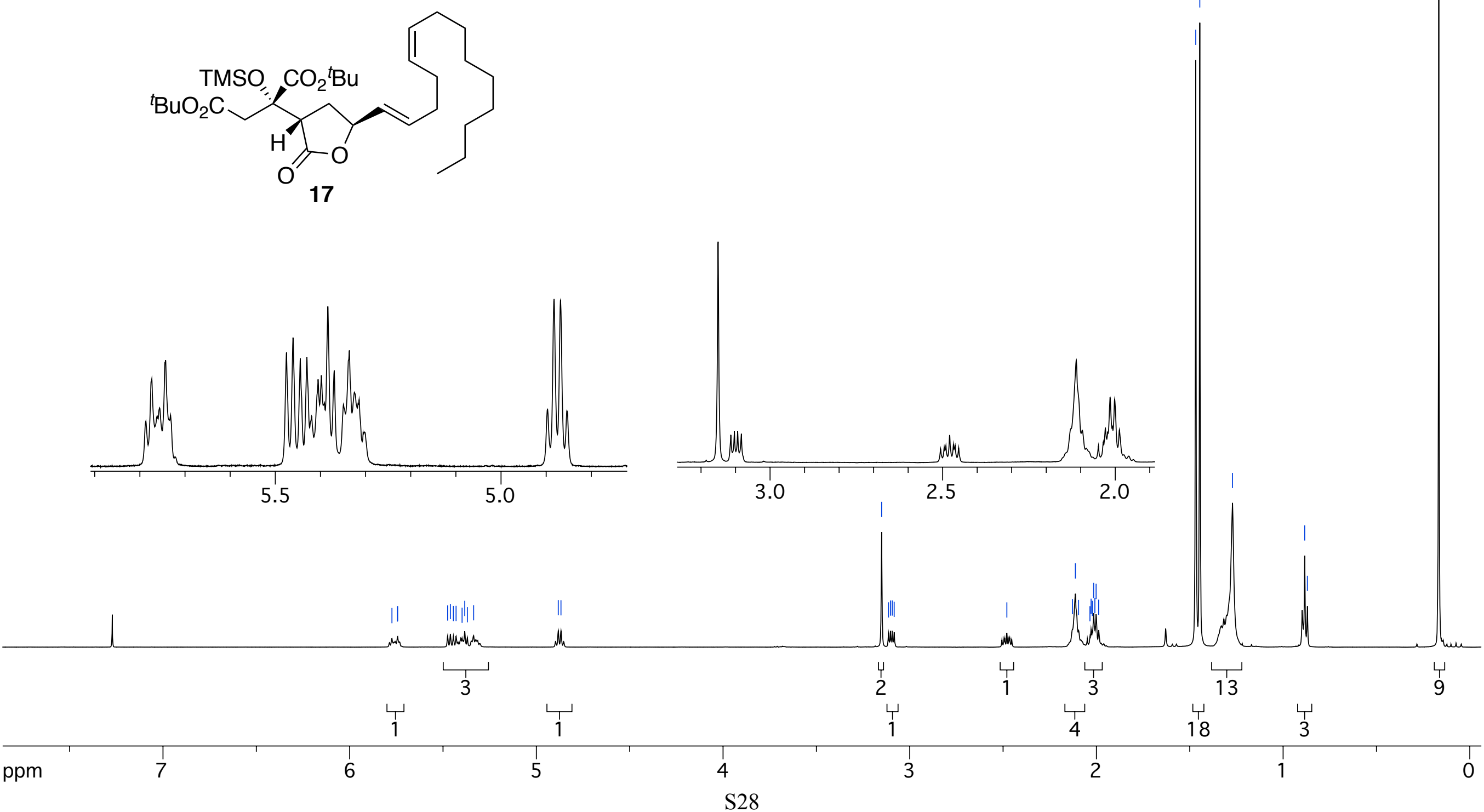

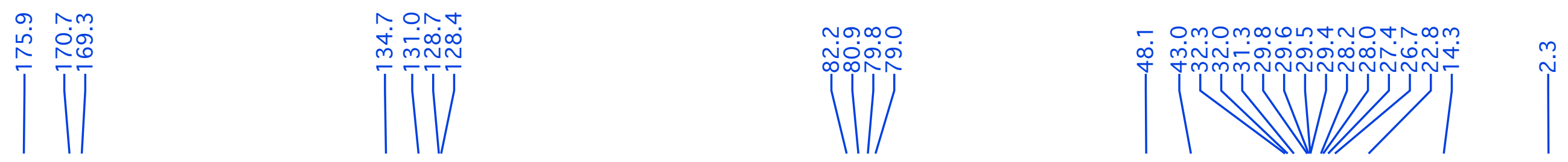

${ }^{13} \mathrm{C}$ NMR spectrum (125 MHz, $\mathrm{CDCl}_{3}$ )
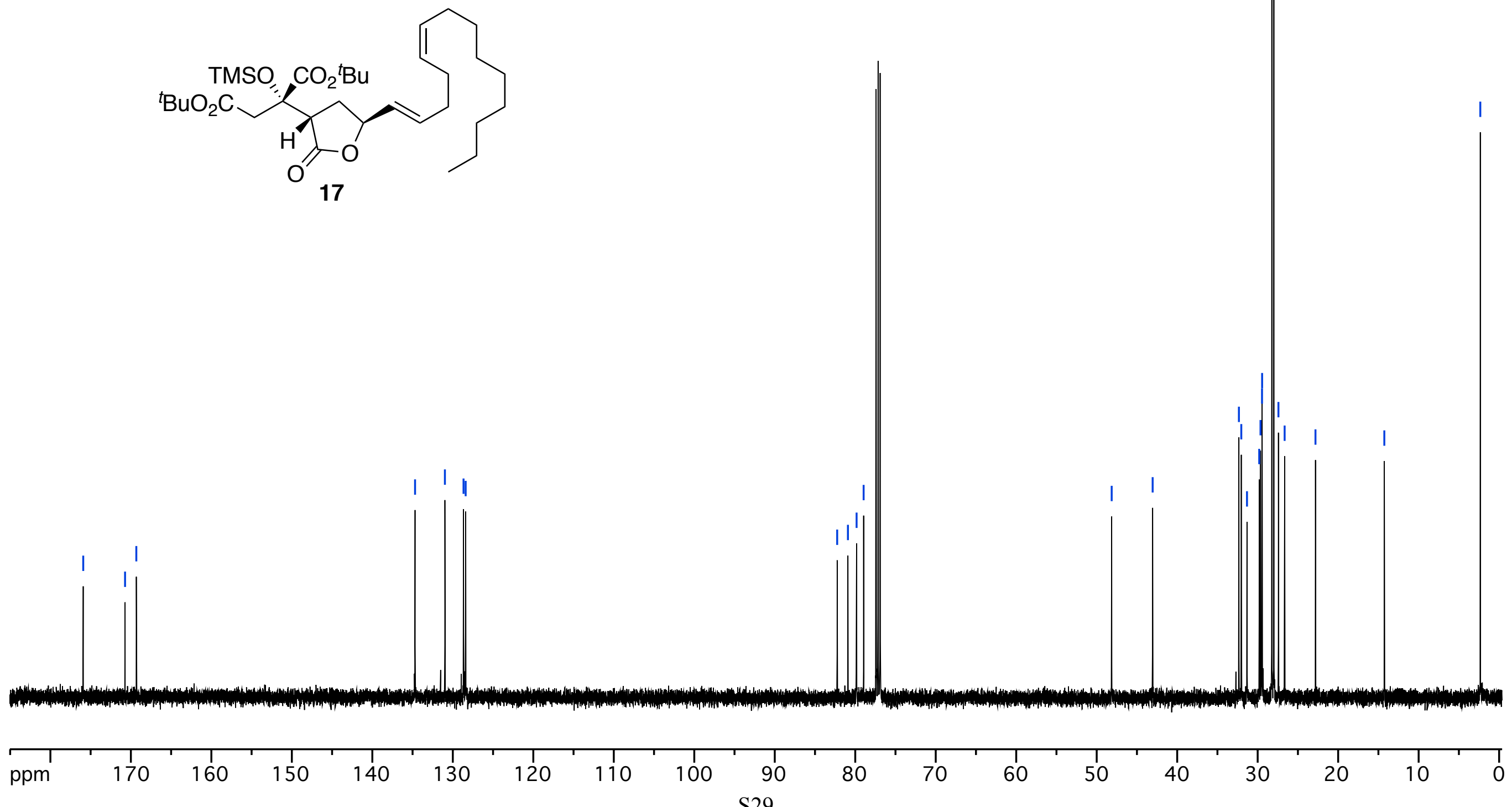
TmNOTNNNOOMmNNNNT- -

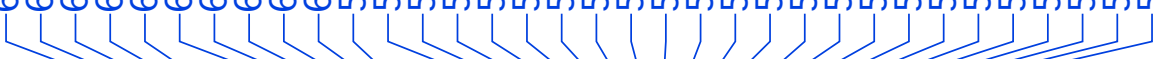

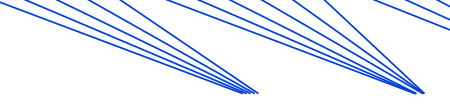

${ }^{1} \mathrm{H}$ NMR spectrum $\left(600 \mathrm{MHz}, \mathrm{CDCl}_{3}\right)$
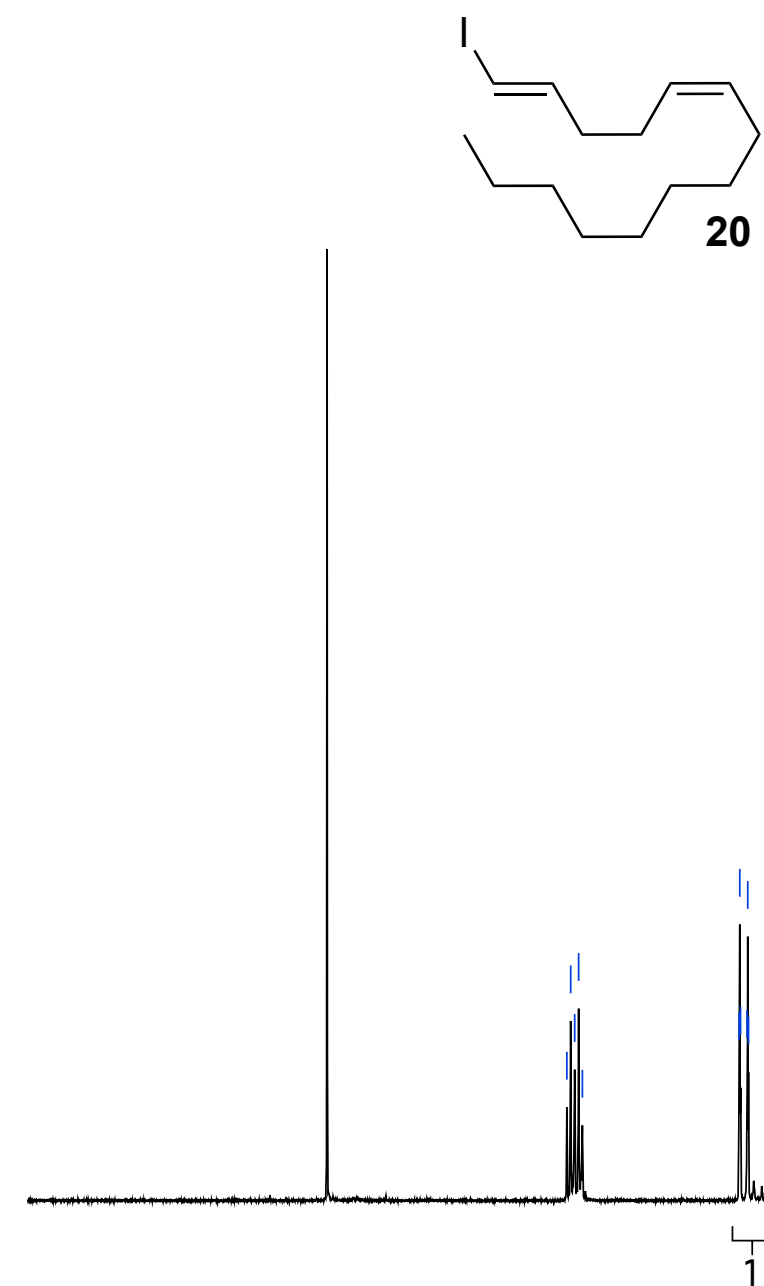

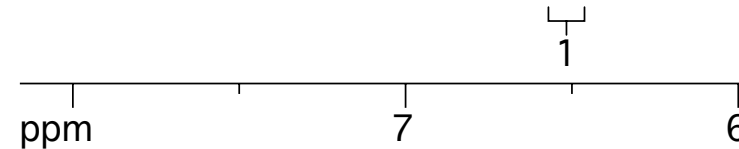

6
0

ppm

7

4

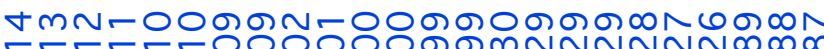
(
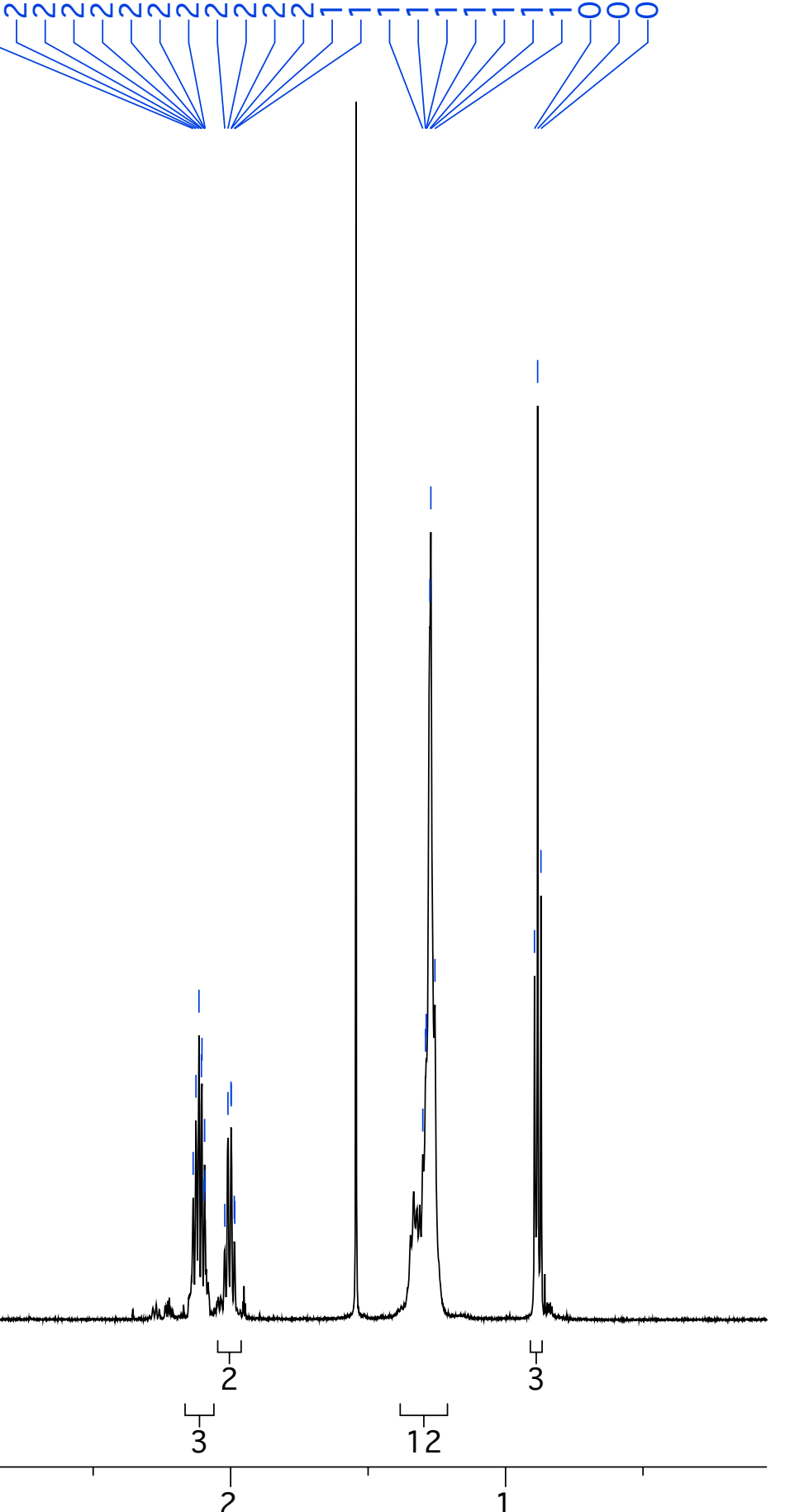
${ }^{13} \mathrm{C}$ NMR spectrum (100 $\mathrm{MHz}, \mathrm{CDCl}_{3}$ )

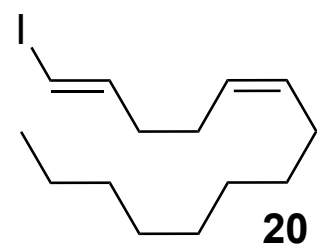




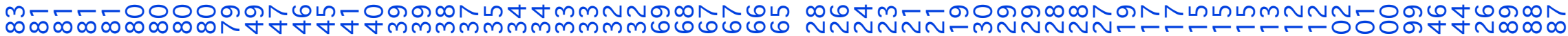
作

${ }^{1} \mathrm{H}$ NMR spectrum $\left(600 \mathrm{MHz}, \mathrm{CDCl}_{3}\right)$
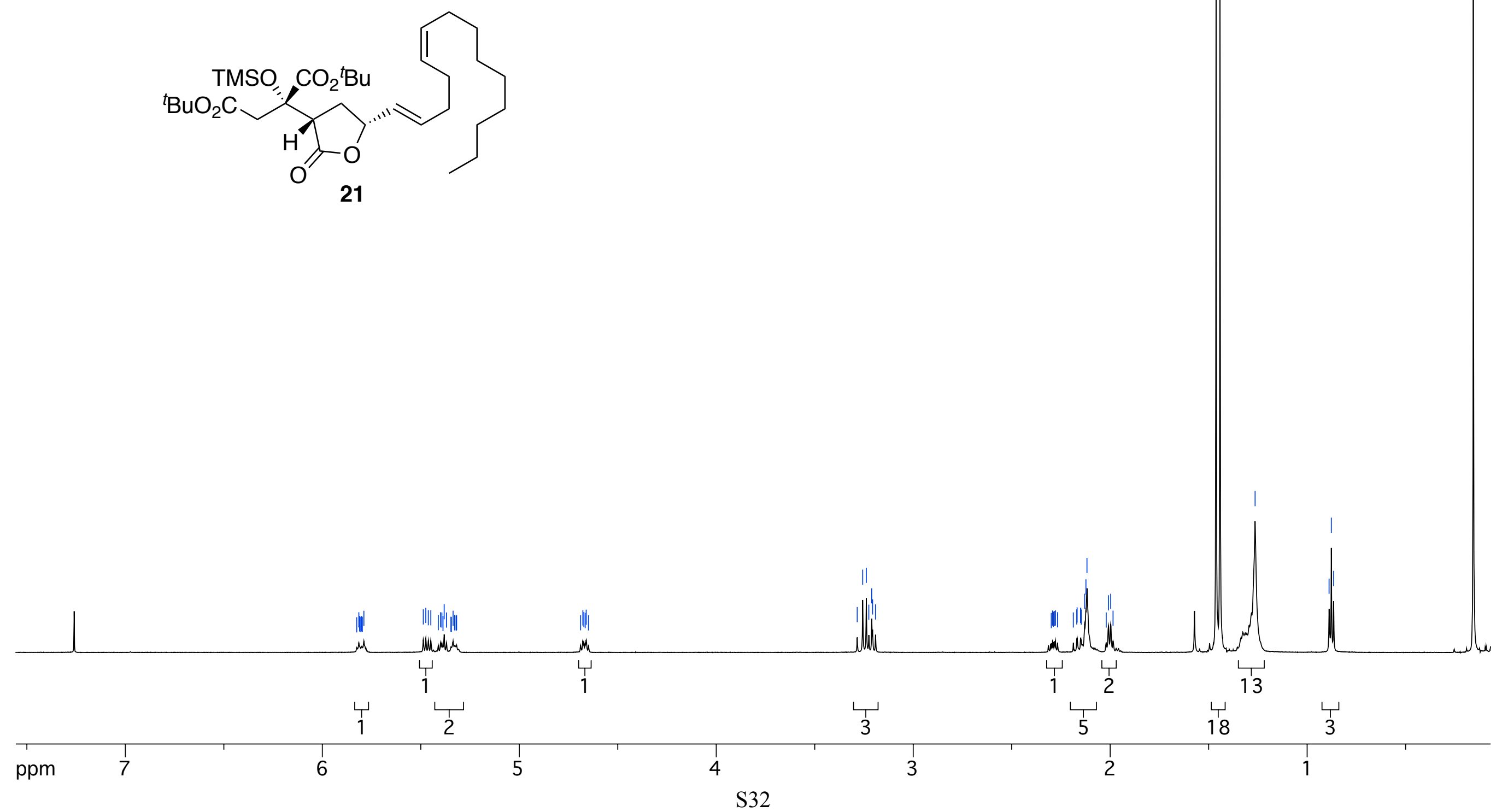

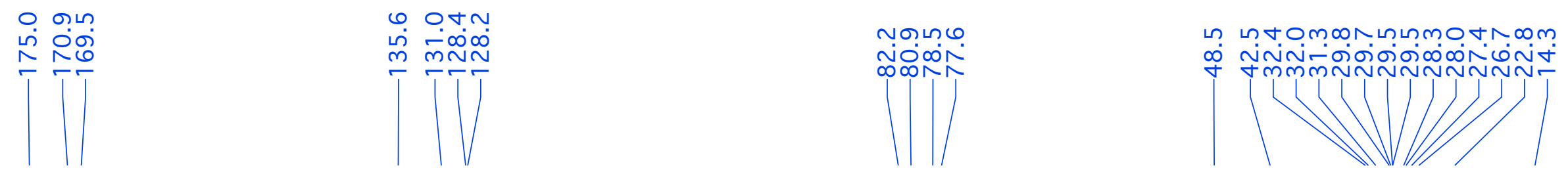

${ }^{13} \mathrm{C}$ NMR spectrum (151 $\mathrm{MHz}, \mathrm{CDCl}_{3}$ )
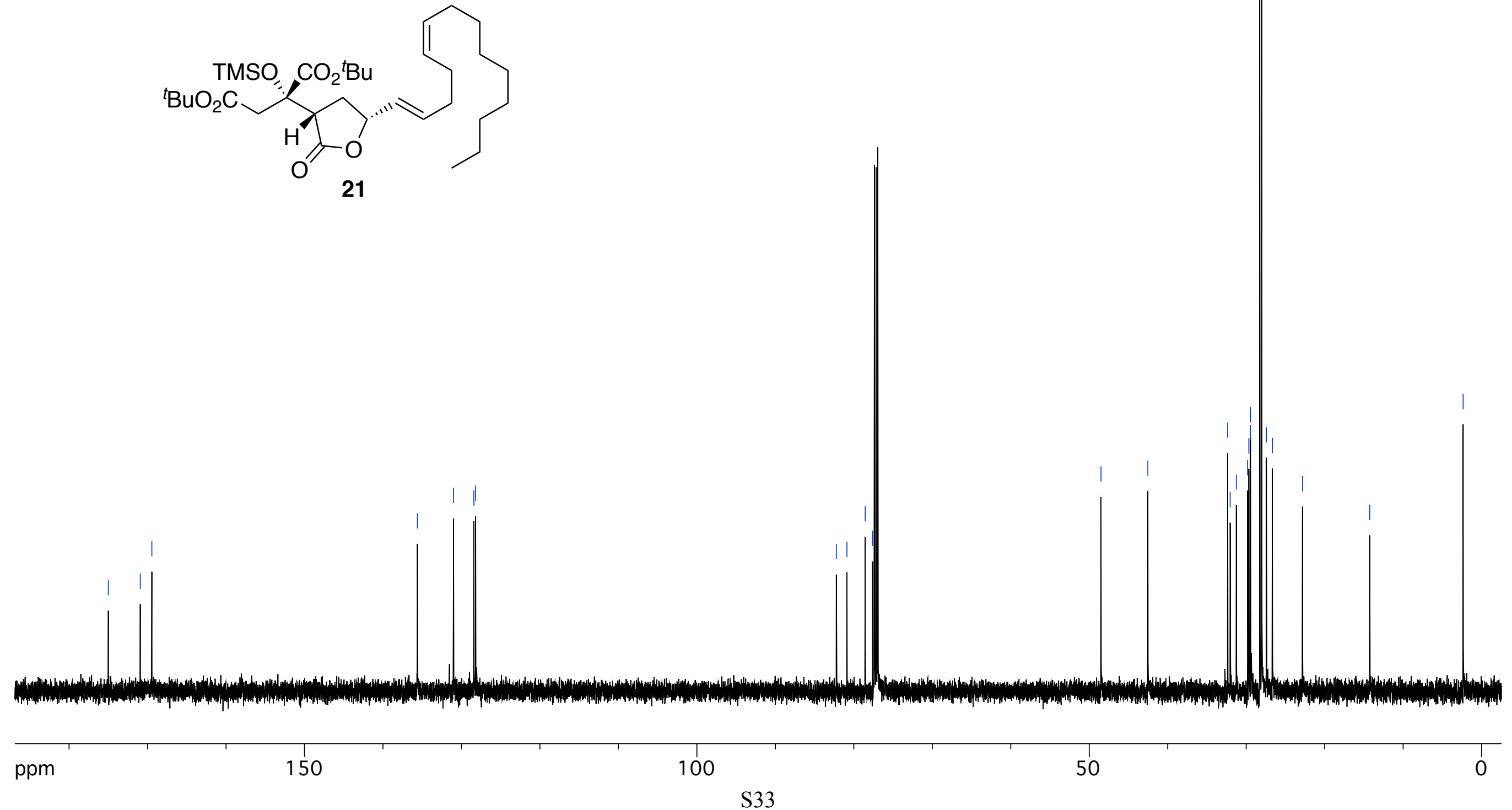

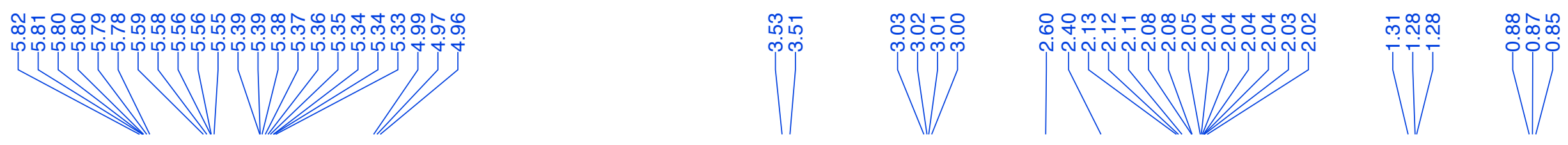

${ }^{1} \mathrm{H}$ NMR spectrum $\left(600 \mathrm{MHz}, d_{6}\right.$-acetone)<smiles>CCCCCCCC/C=C\CC/C=C/[C@H]1C[C@H](C(O)(O)CC(=O)O)C(=O)O1</smiles>

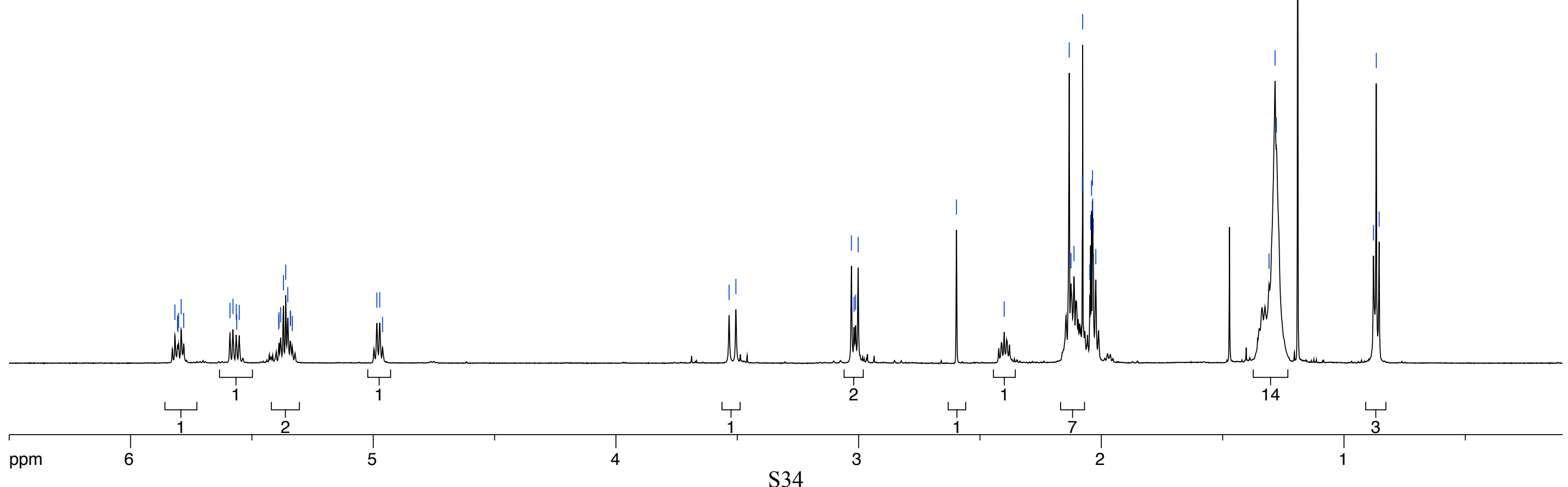

S34 
${ }^{1} \mathrm{H}$ NMR spectrum $\left(600 \mathrm{MHz}, \mathrm{CD}_{3} \mathrm{CN}\right)$

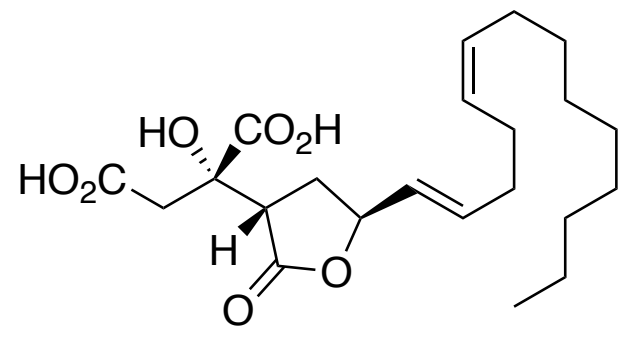

ent-3
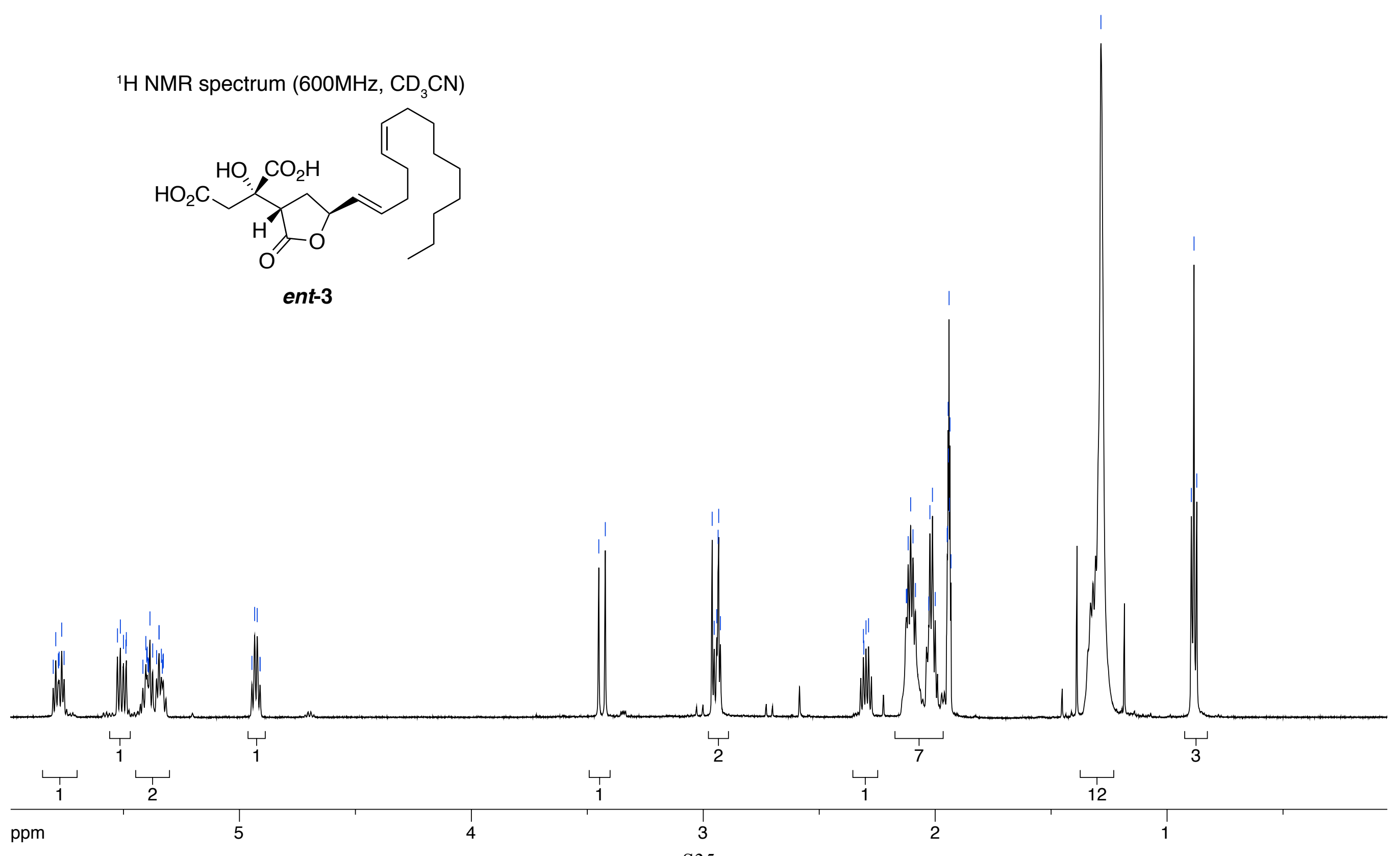

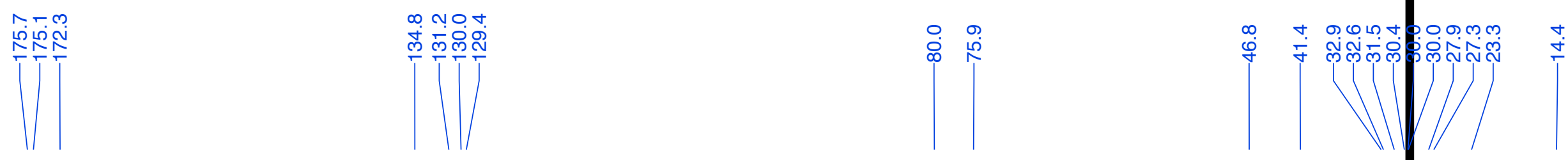

${ }^{13} \mathrm{C}$ NMR spectrum (151 MHz, $d_{6}$-acetone)<smiles>CCCCCCCC/C=C\CC/C=C/[C@H]1C[C@H]([C@](O)(CC(=O)O)C(=O)O)C(=O)O1</smiles> 
${ }^{13} \mathrm{C}$ NMR spectrum (151 MHz, CD $\left.\mathrm{CN}\right)$<smiles>CCCCCCCC/C=C\CC/C=C/[C@H]1C[C@@H]([C@@](O)(CC(=O)O)C(=O)O)C(=O)O1</smiles>

ent-3

\section{.}

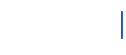

। 
${ }^{1} \mathrm{H}$ NMR spectrum $\left(500 \mathrm{MHz}, \mathrm{CDCl}_{3}\right)$

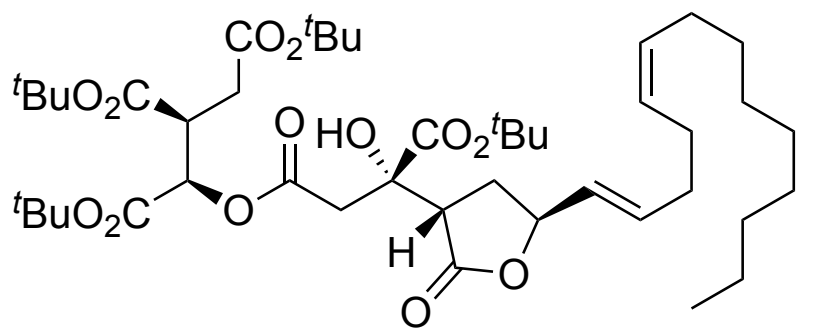

19 Citrafungin A tetra-t-butyl ester
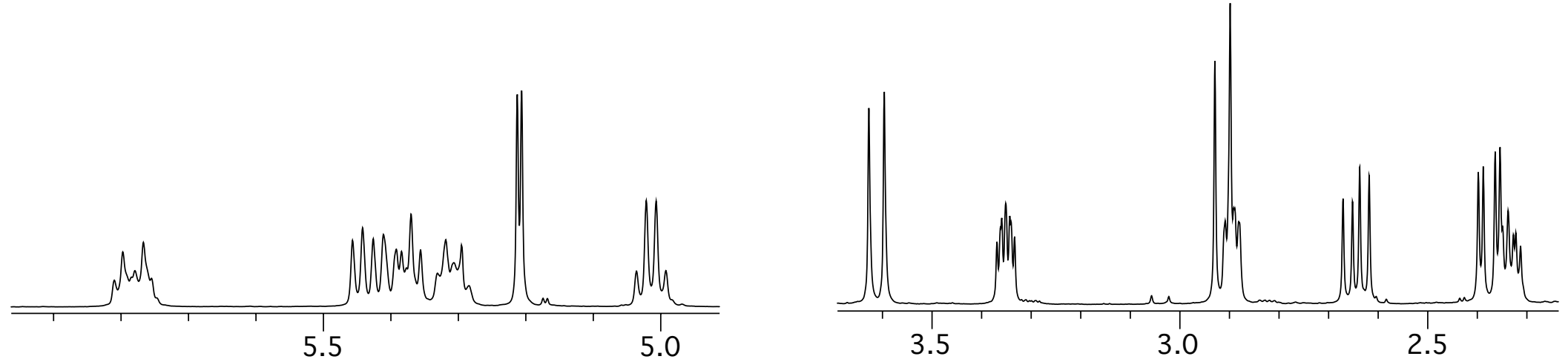

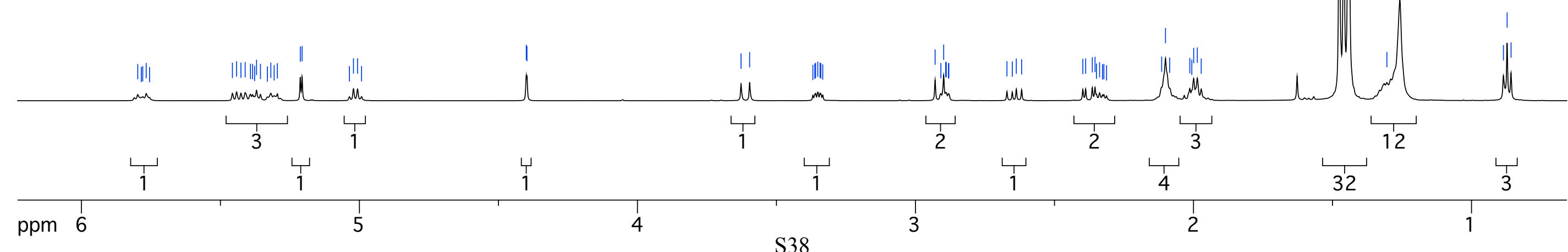


${ }^{13} \mathrm{C}$ NMR spectrum (125.5 $\mathrm{MHz} \mathrm{CDCl}_{3}$ )

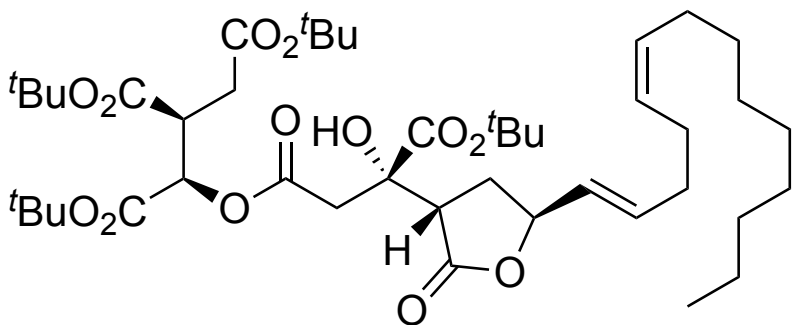

19 Citrafungin A tetra-t-butyl ester 
${ }^{1} \mathrm{H}$ NMR spectrum $\left(500 \mathrm{MHz}, d_{6}\right.$-acetone $)$

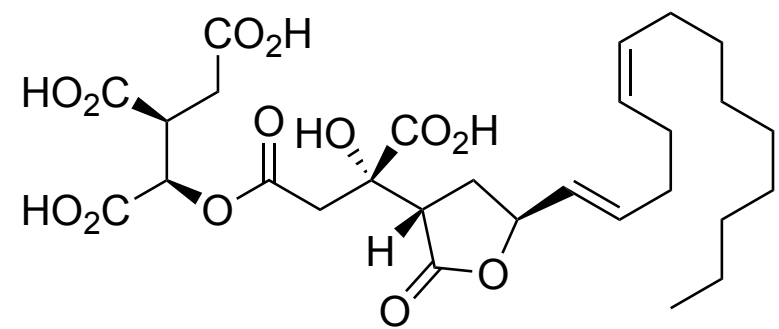

1a Citrafungin $A$

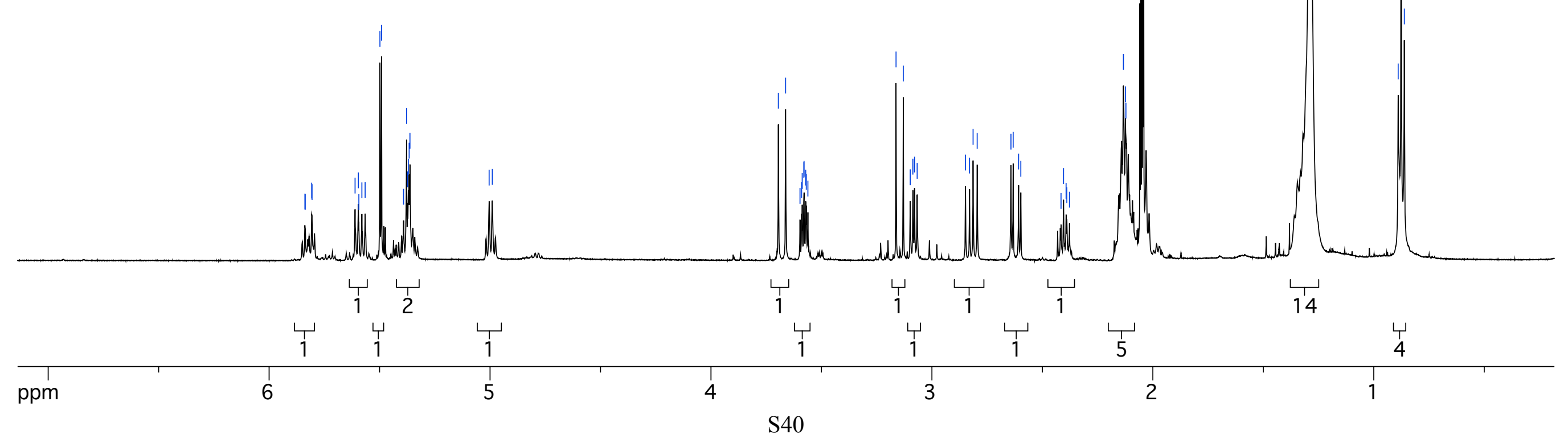



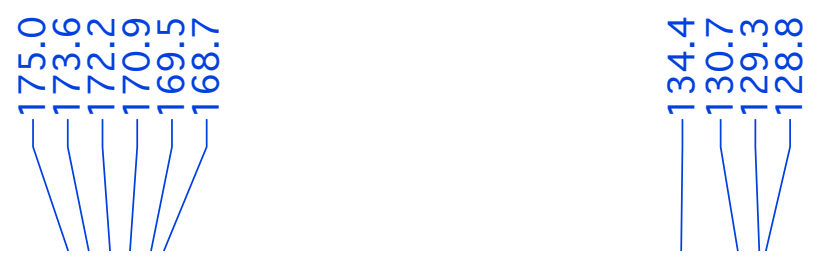

${ }^{13} \mathrm{C}$ NMR spectrum (125.5 MHz, $d_{6}$-acetone)

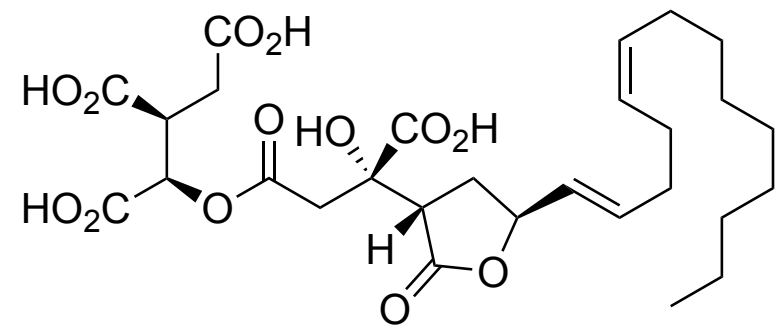

1a Citrafungin $A$

Most downfield signal set to $175.0 \mathrm{ppm}$

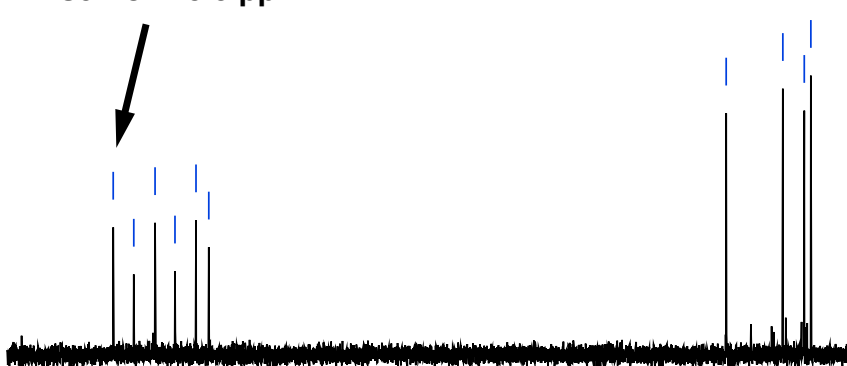

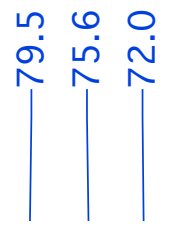

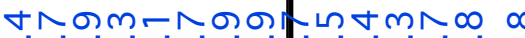

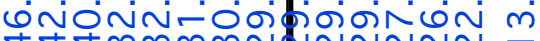

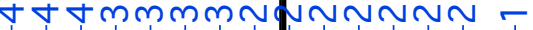

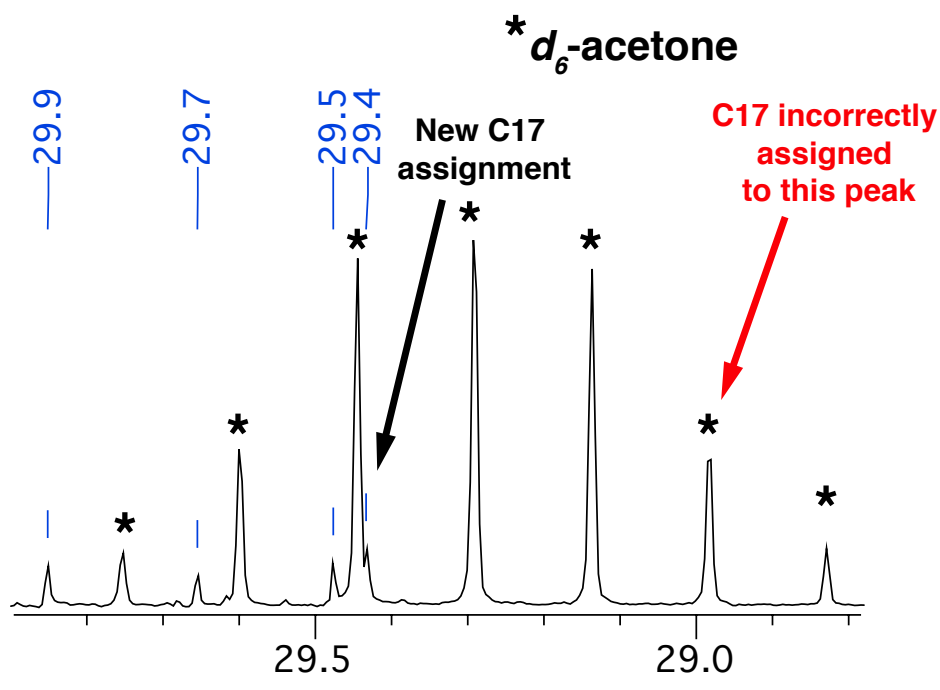




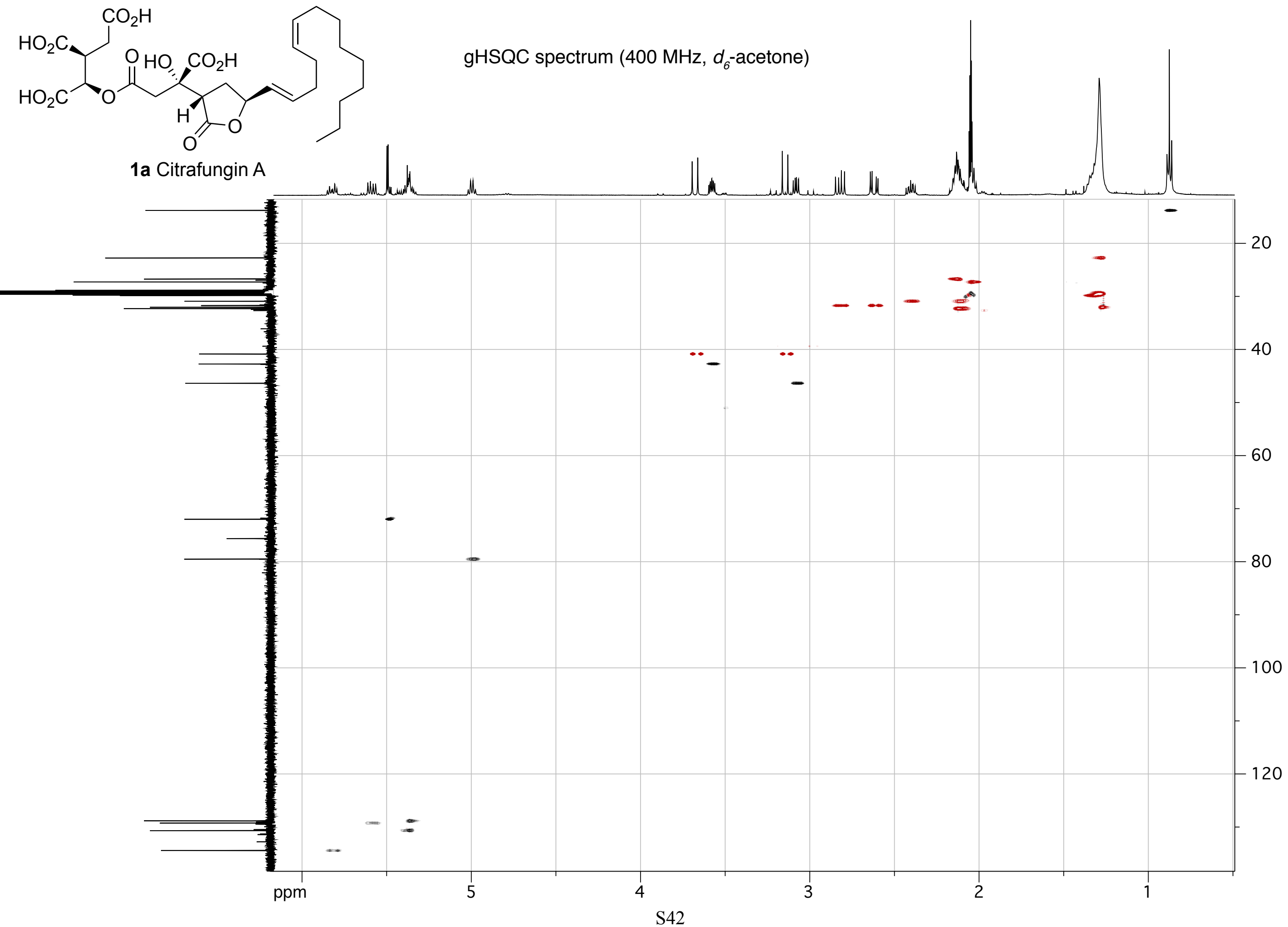




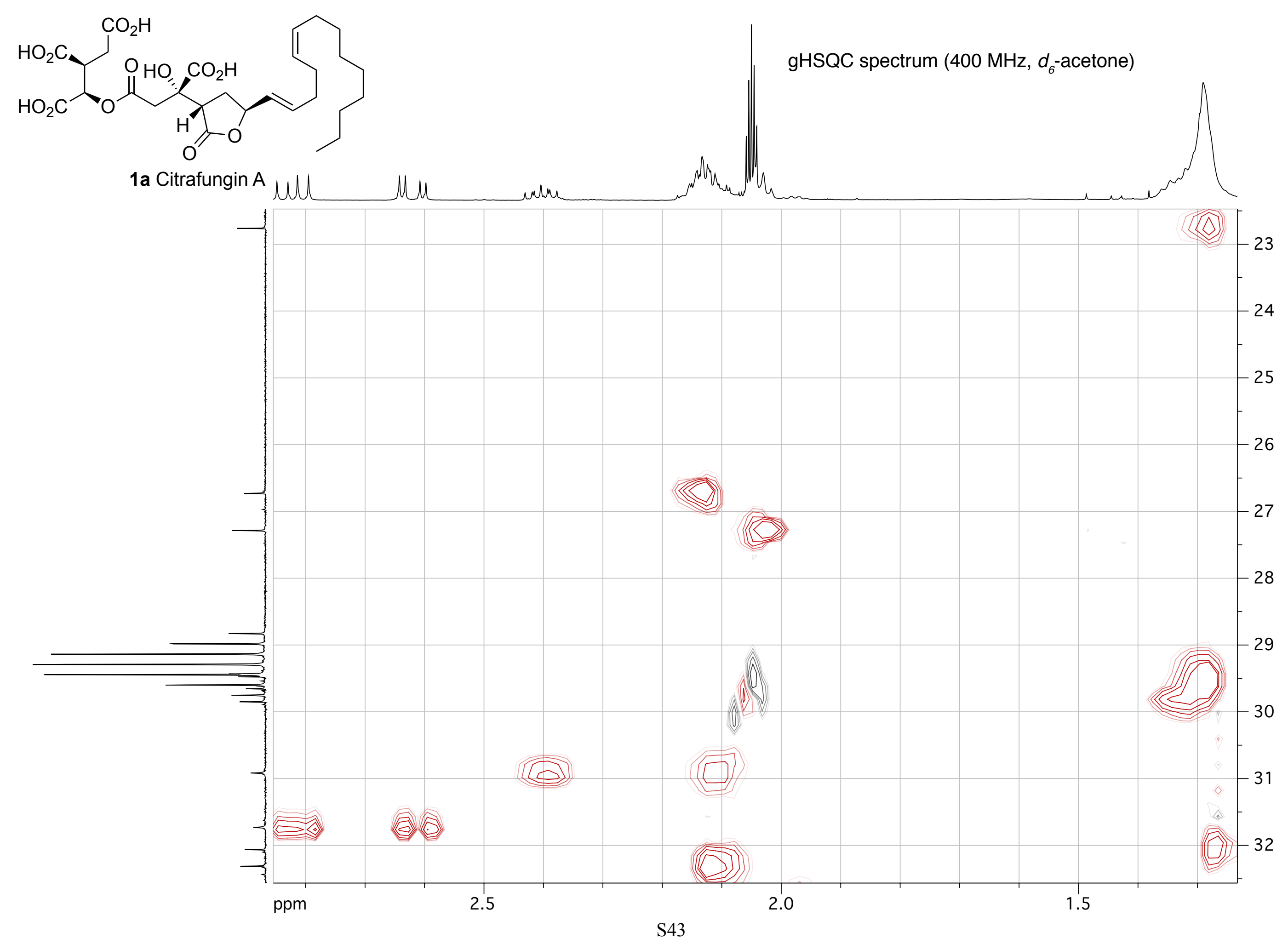

